\title{
A boundary algebraic formulation for plane strain elastodynamic scattering
}

\author{
J. Poblet-Puig*1 and A.V. Shanin ${ }^{\dagger 2}$ \\ ${ }^{1}$ Laboratori de Càlcul Numèric, E.T.S. d'Enginyers de Camins, Canals \\ i Ports de Barcelona, Universitat Politècnica de Catalunya \\ ${ }^{2}$ Department of Physics (Acoustics Division), Moscow State University
}

January 16, 2018

\begin{abstract}
Solving of elastodynamic problems arises in many scientific fields such as wave propagation in the ground, non-destructive testing, vibration design of buildings, or vibroacoustics in general. An integral formulation based on boundary algebraic equations is presented here. This formulation leads to a numerical method with a discretised boundary. An important advantage of the method over the standard boundary element method (BEM) is that no contour (2D) or surface (3D) integral needs to be computed. This feature is helpful in order to obtain a discrete version of the combined field integral equations (designed to damp numerically the fictitious eigenfrequencies) without difficulties caused by the evaluation of hypersingular integrals. The key aspects are: (i) the approach deals with discrete equations from the very beginning; (ii) discrete (instead of continuous) tensor Green's functions are considered (the methodology to evaluate them is demonstrated); (iii) the boundary must be described by means of a regular square grid. In order to overcome the drawback of this third condition the boundary integral is coupled, if needed, with a thin layer of finite elements. This improves the description of curved geometries and reduces numerical errors. The properties of the method are demonstrated by means of numerical examples: the scattering of waves by objects and holes in an unbounded elastic medium, and an interior elastic problem.
\end{abstract}

Keywords: elastodynamics, scattering, boundary integral method

*correspondence: UPC, Campus Nord B1, Jordi Girona 1, E-08034 Barcelona, Spain, e-mail: jordi.poblet@upc.edu

${ }^{\dagger}$ correspondence: 119992, Leninskie Gory, Moscow, Russia, e-mail: a.v.shanin@gmail.com 


\section{List of symbols}

$\begin{array}{ll}b_{j, m}^{v, \eta} & \text { boundary residue of operator } L^{\prime} \\ B A E & \text { Boundary Algebraic Equations } \\ B E M & \text { Boundary Element Method } \\ C F I E & \text { Combined-Field Integral Equations } \\ F E M & \text { Finite Element Method } \\ f_{j}^{\eta} & \text { force applied at node } j, \eta=x, y \\ G_{j, m}^{v, \eta} & \text { tensor Green's function at node } j \text { with source placed at } m \\ h & \text { grid spacing and finite element size } \\ H_{n}^{(1)}, H_{n}^{(2)} & \text { First/Second Hankel function of order } n \\ \mathrm{i} & \text { imaginary unit } \\ j & \text { node of a grid with coordinates }\left(j_{x}, j_{y}\right) \\ L, L^{\prime} & \text { linear operators } \\ R & \text { radius } \\ u^{x}, u^{y} & \text { displacements in the } x \text { and } y \text { directions } \\ v_{P} & \text { P-wave velocity } \\ v_{S} & \text { S-wave velocity } \\ x & \left.\text { coordinate (direction of } j_{x}\right) \\ y & \text { coordinate (direction of } j_{y} \text { ) } \\ \alpha, v, \eta, \tau & \text { superscripts that loop on } x, y \\ \beta, \beta^{\prime} & \text { coefficients of the liner operator } \\ \delta_{j, m} & \text { Dirac delta } \\ \lambda, \mu & \text { Lamé constants } \\ \nu & \text { Poisson's Ratio } \\ \zeta & \text { combination parameter for the CFIE } \\ \omega & \text { pulsation of the problem } \\ \Omega, \Omega^{\prime} & \text { domains } \\ \rho & \text { volume density of the solid domain } \\ \odot_{i} & \text { Set of nodes linked to the node } i \text { (and also the node } i \text { ) }\end{array}$

\section{Introduction}

Solving of elastodynamic problems has important applications in different fields of science and technology. Among other examples: propagation of vibrations in the ground and the effect on building comfort [18,9], non-destructive testing and detection of fractures and cracks $[16,15]$ or seismic wave propagation [7].

Numerical solution of exterior problems possesses difficulties related to domain truncation, dispersion and pollution (non-local deterioration of the numerical solution due to the increase of the problem frequency which leads to numerical solutions with wavenumbers differing from the exact one) effects [5], which always deteriorate the quality of the result. A frequently considered technique that overcomes mainly the first of these difficulties is the boundary element method (BEM, [3, 32, 39, 26]).

A less popular family of methods are those dealing with a discrete version of the equations from the very beginning and reducing the problem to the boundary. Among these methods, we should mention boundary algebraic equations (BAE) for the 
potential [29] or wave propagation (scalar Helmholtz equation) problems [48, 4, 35, 36]. They have an important property that no boundary integral needs to be computed. This enables one to avoid evaluation of hypersingular integrals at the boundary, which is a major issue of BEM. Also, the difference potential method has been successfully applied to a scalar Helmholtz equation [30].

The methods used to study diffraction by crystallographic and material lattices of different shapes at microscopy level also possess some similarity with the general purpose discrete methods mentioned above. See for example [42, 43, 44].

Most of these alternative methods are used to solve the Laplace or scalar Helmholtz equation. Only a few applications to solid mechanics and elastodynamics can be found. See for example the application of the method of difference potentials to linear elastic fracture mechanics in [50].

The aim of the present work is to extend the method presented in $[35,36]$ to the elastodynamic scattering in solids. To the best of the authors knowledge, BAE have not been used before in elastodynamics. An external excitation problem is studied, i. e. there exists a homogeneous elastic space with a finite cavity. The field is excited by a force applied to the boundary of the cavity. It is necessary to find the field radiated into the space. The radiated field can be computed in quadratures by the Green's formula when the displacement of the boundary of the cavity is found. So, our aim is to to find the displacement of the boundary.

Virtually, the infinite elastic outer space is meshed, and the governing equations are approximated on the mesh using the finite element method (FEM). So, the radiation problem is solved from the very beginning for the discretized problem. The mesh (see Fig. 4) consists of a an infinite regular part $\Omega_{\text {ext }}$ covering almost all the space and a thin irregular layer $\Omega_{\text {int }}$ placed to match the boundary of the cavity $\Gamma_{\text {int }}$ with the regular mesh. The boundary between $\Omega_{\text {int }}$ and $\Omega_{\text {ext }}$ will be denoted by $\Gamma_{\text {ext }}$. For simplicity we assume that $\Gamma_{\text {ext }}$ and $\Gamma_{\text {int }}$ have no common nodes. Although mathematically two parts of the mesh work similarly, they are treated numerically in a different way. The FEM formalism is developed only for the irregular mesh, while the regular mesh is used to provide a radiation condition for the latter FEM model on the surface $\Gamma_{\text {ext }}$.

The radiation condition has form of a matrix linking the forces and the displacements on the interface $\Gamma_{\text {ext }}$. Thus, the method is close to the boundary element method (BEM) on $\Gamma_{\text {ext }}$. However, unlike BEM, our method does not require integration of hypersingular kernels on the surface. Instead, the matrix is constructed explicitly by using the Green's function of the regular mesh.

The new method is close to BEM, so we can compare the advantages and disadvantages of both. The memory requirements are similar (of order of the number of nodes on the surface of the cavity). But the new method does not require integration of singular functions on the surface of the cavity, which is a considerable advantage. Here we make difference between the integration and the summation over the nodes of the mesh, which is of course a discrete analogue of integration. We take into account that for the integration one should introduce a smooth parametrization of the surface and a dense quadrature point set inside each boundary element. This procedure limits the efficiency of BEM.

A drawback of BEM is that the boundary integrals must be computed quite accurately. Otherwise, large errors in the numerical solution can be found as reported 
in [47]. There, the analysis is focused on the acoustic scattering problem (scalar Helmholtz equation) and the CHIEF technique [41] is used in order to damp the fictitious eigenfrequencies. CHIEF requires the consideration of some colocation point outside the problem boundary respecting the type of integrals involved in the formulation.

An usual alternative to CHIEF technique is to consider a Burton-Miller formulation [6] in order to derive a combined field integral equation (CFIE) which is also free of fictitious eigenfrequencies. CFIE requires a modification of the boundary integral form: by means of the derivatives of the original equation. The modified boundary integral is free of fictitious eigenfrequencies with the need of computing hypersingular integrals (originated due to the derivative of the original equation). Their precise evaluation is even more critical and the consequences to the quality of the BEM solution are more important.

All these aspects commented and reported for the scalar Helmholtz equation also appear in the elastodynamic problem. We understand the proposed BAE method as a valid option to consider a CFIE formulation without the need to deal with the integration of singular kernels in the boundary because these integrals simply do not exist. Consequently, BAE eliminates this source of error.

We should note that the new method comprises a computation of the Green's function of the regular mesh, which is also a time consuming procedure. In the current realization of the method this step is done by using complex contour integration. Therefore, it is not easy to compare the new method and BEM in terms of efficiency, but we believe that they are close to each other. Both methods admit optimization. Some important works on fast multipole BEM techniques (see for example [7]) have been done in order to improve the efficiency of BEM. Our method can be optimized by using known asymptotics of the discrete Green's function [28]. Both methods are immune to spurious resonances since they share the same Burton-Miller or CFIE technique to avoid this.

In view of practical numerical computations, we believe that the main advantage of the new method is its simplicity and universality. The tabulation of the Green's function on a regular mesh can be implemented as a standard and optimized routine, and all the rest is a FEM implementation on a relatively small mesh. Any peculiarities of the scatterer can be modeled in known ways (for example, crack tips can be modeled by singular finite elements [46] or some modern techniques).

Considering the exposed characteristics, the BAE method applied to elastodynamics is more indicated for those situations where the geometry of scatterers or holes in the domain are simple enough to be described by means of a regular grid (i.e. rectangles, L-shaped). In that geometries all the potentialities of BAE (lack of boundary integrals, straightforward use of CFIE formulation to damp fictitious eigenfrequencies and proper representation of unbounded domains) are present with minor drawbacks. For curved and complex geometries the combination with other techniques (i.e. coupling with FEM commented in Section 2.6.2) is required. And when the exact representation of the boundary geometry is the key aspect, advanced interpolation techniques such as isogeometric analysis and the use of CAD and nurbs [45] is most probably a much better alternative to BAE.

Finally, the fact that BAE is based on a structured grid and linear algebraic 
equations are directly obtained through loops on boundary nodes makes the implementation to be quite straightforward. The main differences with respect to BEM is that all integration routines are not needed in BAE and that discrete Green's functions are used instead of the continous ones. Since the formulation is discrete from the very beginning, it allows to perform in an algebraic way operations such as the derivation or the computation of the equivalent of the BEM surface traction tensor. This makes much more easy the derivation of equations to code and the implementation itself. So, for someone who needs a straightforward solution of the problem with a simple geometry such as a cube or other grid-shaped boundary avoiding the specific integration routines for singular kernels and large analytical derivations of operators from the continuous Green's function, the proposed method is a clear and valid alternative.

The structure of the paper is as follows. In Section 2 the new method is presented. It includes the discrete formulation of the two-dimensional plane strain elastodynamic problem, the evaluation of the tensor discrete Green's function, and some implementation details. Section 3 contains numerical examples. The formulation and examples are two-dimensional. Minor modifications are required in order to make the formulation and ideas in Sections 2.2 and 2.3 three-dimensional (implementation and computational efficiency is different step).

\section{Methodology}

\subsection{Problem statement for plane strain elastodynamics}

Consider a system of two-dimensional plane strain elastodynamic equations (NavierCauchy equations of motion, see for example $[2,10])$ :

$$
\begin{gathered}
(\lambda+2 \mu)\left(\frac{\partial^{2} u^{x}}{\partial x^{2}}+\frac{\partial^{2} u^{y}}{\partial x \partial y}\right)+\mu\left(\frac{\partial^{2} u^{x}}{\partial y^{2}}-\frac{\partial^{2} u^{y}}{\partial x \partial y}\right)=-\rho \omega^{2} u^{x}+f_{v}^{x} \\
(\lambda+2 \mu)\left(\frac{\partial^{2} u^{x}}{\partial x \partial y}+\frac{\partial^{2} u^{y}}{\partial y^{2}}\right)+\mu\left(-\frac{\partial^{2} u^{x}}{\partial x \partial y}+\frac{\partial^{2} u^{y}}{\partial x^{2}}\right)=-\rho \omega^{2} u^{y}+f_{v}^{y}
\end{gathered}
$$

where $\lambda$ and $\mu$ are the Lamé's constants, $\rho$ is the volume density, $\left(f_{v}^{x}, f_{v}^{y}\right)$ is the external force per unit volume, and $u^{x}, u^{y}$ are the displacements in the $x$ and $y$ directions. Time-harmonic dependence of the form $e^{-i \omega t}$ is omitted hereafter, with the pulsation of the problem $\omega$ is $2 \pi$ times the frequency.

Assume that parameter $\omega^{2}$ has a small positive imaginary part. This corresponds to a small absorption in the medium. Thus, seek the radiated wave as an exponentially decaying field, i. e. apply the limiting absorption principle [49, 38]. Of course, one can formulate the standard radiation condition for real $\omega^{2}[22,11,19,17]$, but this makes the consideration slightly more complicated. All statements of the paper remain valid for real $\omega^{2}$.

\subsection{Problem formulation}

As it has been proposed for the scalar Helmholtz equation in [36], we consider a cubic (three-dimensional) or rectangular (two-dimensional) grid of size $h$ in the problem 


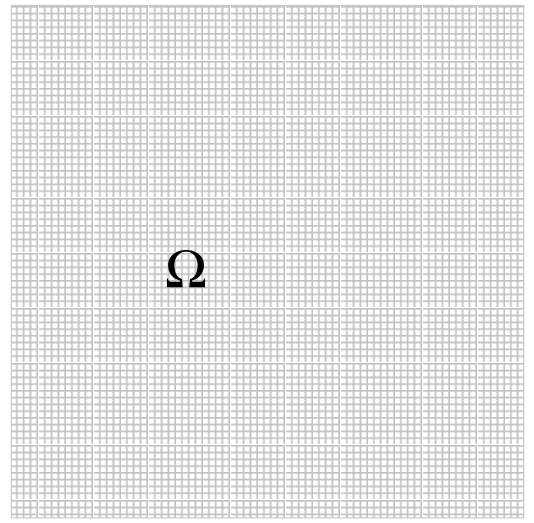

(a)

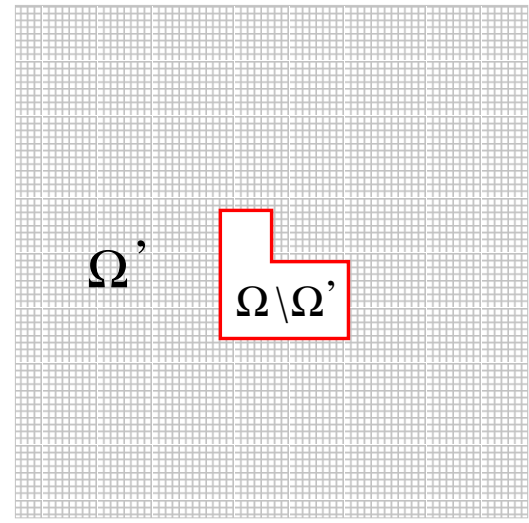

(b)

Figure 1: Domains involved in the formulation: (a) unbounded without scatterer $\Omega$; (b) unbounded but truncated with boundary $\partial \Omega^{\prime}$ around the scatterer. All of them are subsets of $h \mathbb{Z}^{2}$, with grid size $h$.

domain $\Omega^{\prime}$, see Fig. $1 . \Omega^{\prime}$ is a part of the whole two- or three-dimensional space $\Omega$. Both are subsets of $h \mathbb{Z}^{n}$, with grid size $h$. The case $n=2$ is considered because both the formulation and the examples shown here are two-dimensional. However, the idea could be applied to three-dimensional problems $n=3$ (with small modifications in the formulation and more efforts required in order to obtain the three-dimensional discrete Green's function and to make a computationally efficient implementation). The nodes are denoted by the index $j$ with grid coordinates $\left(j_{x}, j_{y}\right)$.

We call two nodes neighbours if the nodes belong to the same finite element (elementary square in $2 \mathrm{D}$ or cube in $3 \mathrm{D}$ ) of the full mesh $\Omega$.

Define the boundary of the scatterer $\partial \Omega^{\prime}$ as the set of all neighbours of the nodes belonging to $\Omega \backslash \Omega^{\prime}$ in $\Omega^{\prime}$. Nodes of $\Omega^{\prime} \backslash \partial \Omega^{\prime}$ will be called the internal nodes of $\Omega^{\prime}$.

In the tensor notations, the subscripts are used to indicate the node of the grid. The superscripts indicate the components (polarization of the field). Sum over repeated indices is always assumed.

Define linear operators $L$ and $L^{\prime}$ on the meshes $\Omega$ and $\Omega^{\prime}$, respectively. The definition is as follows:

$$
\begin{gathered}
L[u]_{j}^{\alpha} \equiv \sum_{q \in \Omega} \beta_{j, q}^{\alpha, \eta} u_{q}^{\eta}, \quad j \in \Omega, \quad \alpha, \eta=x, y, \\
L^{\prime}[u]_{j}^{\alpha} \equiv \sum_{q \in \Omega^{\prime}} \beta_{j, q}^{\prime \alpha, \eta} u_{q}^{\eta}, \quad j \in \Omega^{\prime}, \quad \alpha, \eta=x, y,
\end{gathered}
$$

$x$ and $y \in h \mathbb{Z}$; and $L[u]_{j}^{\alpha}, L^{\prime}[u]_{j}^{\alpha}$ and $u_{q}^{\eta} \in \mathbb{C}$. All these operators approximate the Navier-Cauchy equations (1) with traction-free boundary conditions, each in its domain because the coefficients $\beta$ are obtained by means of a proper discretisation of the equations using finite elements, finite differences or similar (see below).

Matrices of the coefficients $\beta_{j, q}^{\alpha, \eta}, \beta_{j, q}^{\alpha, \eta}$ must have the following properties:

1. the elements $\beta_{j, q}^{\alpha, \eta}, \beta_{j, q}^{\prime \alpha, \eta}$ are equal to zero if $q$ and $j$ are not neighbours; 
2. matrices are symmetrical, i.e.

$$
\beta_{j, q}^{\alpha, \eta}=\beta_{q, j}^{\eta, \alpha}, \quad \beta_{j, q}^{\alpha, \eta}=\beta_{q, j}^{\prime \eta, \alpha}
$$

for all admissible $j$ and $q$;

3. operator $L^{\prime}$ is a restriction of $L$ in the following specific sense:

$$
\beta_{j, q}^{\prime \alpha, \eta}=\beta_{j, q}^{\alpha, \eta} \quad \text { if } j, q \in \Omega^{\prime}, \quad j \text { or } q \notin \partial \Omega^{\prime}
$$

The condition means that either $j$ or $q$ is an internal nodes of $\Omega^{\prime}$.

These properties are in general satisfied if the operator is build as an assembly of square finite elements. It is the case of the operator proposed in Appendix A. One of the key aspects of the method is to define the operators such that in the field version or in any of the possible truncations due to the representation of the geometry, the symmetry conditions of the Eq. (5) are satisfied. This must be checked once the operator is defined.

According to the first property all sums in (3), (4) are finite for each $j$. We assume also that matrix $\beta$ is homogeneous with respect to translations along the coordinate lines. This property will enable us to use Fourier transform to compute the Green's function of $L$.

Examples of operator $L$ are provided in Appendix A. The nodes are called edgeadjacent if they are adjacent along one of the coordinate lines, $h$ is the size of the grid. Appendix A details also how the discretisation of an uniformly distributed force per unit volume is done.

\subsection{Derivation of discrete analogue of boundary integral equa- tions}

The inhomogeneous equation which is the discrete representation of Eq. (1) and accounts for possible point or body forces is as follows:

$$
L^{\prime}[u]_{j}^{\alpha}=\sum_{q \in \Omega^{\prime}} \beta_{j, q}^{\prime \alpha, \eta} u_{q}^{\eta}=f_{j}^{\alpha} \quad \forall j \in \Omega^{\prime}, \quad \alpha, \eta=x, y
$$

The solution of Eq. (7) approximates the solution of the Navier-Cauchy equations (Eq. (1)). Since the BAE method is based on a FEM operator, it is expected the numerical result to approximate the solution of the problem according to similar criteria. The solutions of Eq. (7) are in the $L^{2}$ space. The reduction of the element size (h-refinement) leads to quadratic convergence if linear finite elements are considered (error measured by means of the $L^{2}$-norm) [51]. This basic result can be altered in wave based problems (i.e. Helmholtz equation or elastodynamics). A priori error estimators for the Helmholtz equations are provided in [5] and numerical evidences of the convergence in elastodynamics problems are shown in [25].

Based on the limiting absorption principle [49,38], we assume that $\operatorname{Im}[\omega]>0$, we are interested in the limit when $\operatorname{Im}[\omega] \rightarrow 0$ ), that the sources $f_{j}$ are localized in a finite area, and we look for the solution decaying exponentially at infinity. The 
limiting absorption principle is used not only for the theoretical formulation, but also for practical computation of the discrete Green's function.

Let $G_{j, m}^{\alpha, \eta}$ be the discrete Green's function (where a point force is placed at the position $m$ ) of the mesh $\Omega$, i.e. let it be a solution of equation

$$
L\left[G_{m}^{\alpha, \eta}\right]_{j}=\sum_{q \in \Omega} \beta_{j, q}^{\alpha, v} G_{q, m}^{v, \eta}=\delta_{j, m}^{\alpha, \eta}=\delta_{j, m} \delta_{\alpha, \eta}
$$

$\delta_{j, m}$ the Kronecker delta (unit force is placed at node $m$ ). The discrete Green's function provides the displacement field due to a force acting in the grid node $m$

$$
u_{j}^{\alpha}=G_{j, m}^{\alpha, \eta} f_{m}^{\eta} \quad \alpha, \eta=x, y
$$

The Green's function is symmetrical:

$$
G_{q, p}^{v, \tau}=G_{p, q}^{\tau, v}
$$

The proof of the identity is straightforward if the symmetrical property of $\beta$ is taken into account

$$
\begin{aligned}
G_{p, q}^{\tau, v}=\sum_{m \in \Omega} G_{m, q}^{\eta, v} \delta_{m, p}^{\eta, \tau}=\sum_{m \in \Omega} G_{m, q}^{\eta, v} \sum_{n \in \Omega} \beta_{m, n}^{\eta, \alpha} G_{n, p}^{\alpha, \tau}= & \\
& \sum_{n \in \Omega}\left(\sum_{m \in \Omega} G_{m, q}^{\eta, v} \beta_{m, n}^{\eta, \alpha}\right) G_{n, p}^{\alpha, \tau}=\sum_{n \in \Omega} \delta_{q, n}^{v, \alpha} G_{n, p}^{\alpha, \tau}=G_{q, p}^{v, \tau} .
\end{aligned}
$$

Below we need the following property:

$$
\sum_{i \in \Omega^{\prime}} \sum_{j \in \odot_{i}} \beta_{i, j}^{\prime \alpha, \eta} u_{j}^{\eta} w_{i}^{\alpha}=\sum_{j \in \Omega^{\prime}} \sum_{i \in \odot_{j}} \beta_{j, i}^{\prime \eta, \alpha} w_{i}^{\alpha} u_{j}^{\eta}
$$

for any functions $u_{j}^{\eta}$ and $w_{i}^{\alpha}$. Here $\odot_{i}$ indicates that the summation is performed about the neighbours of node $i$ (and node $i$ itself). Eq. (12) is true due to locality and symmetricity of the operator. It is also important to note that the sums over $\Omega^{\prime}$ and over $\left(\odot_{i}\right)$ commute (it is the same to make the sum over all nodes $i \in \Omega^{\prime}$ and then consider the nodes $j$ surrounding $i$, or to make the sum over all nodes $j \in \Omega^{\prime}$ and then consider the nodes $i$ surrounding $j$ ). Consequently, Eq. (12) can be proven by applying the symmetry property of Eq. (5) to the operator in the right-hand-side of the equality and, in addition, commute $w_{i}^{\alpha}$ with $u_{j}^{\eta}$. It can be understood as a discrete analogue of Betti's theorem [1].

The symmetry property of the Green's function in Eq. (10) can also be proven if it is considered in Eq. (12) that $u_{j}^{\eta}=G_{j, q}^{\eta, v}$ and $w_{i}^{\alpha}=G_{i, p}^{\alpha, \tau}$.

Particularizing Eq. (12) for the case the discrete Green's function $G_{i, m}^{\alpha, \tau}$ playing the role of $w_{i}^{\alpha}$, we have

$$
\begin{aligned}
& \sum_{i \in \Omega^{\prime}} L^{\prime}[u]_{i}^{\alpha} G_{i, m}^{\alpha, v}=\sum_{i \in \Omega^{\prime}} \sum_{j \in \odot_{i}} \beta_{i, j}^{\prime \alpha, \eta} u_{j}^{\eta} G_{i, m}^{\alpha, v}= \\
= & \sum_{j \in \Omega^{\prime}} \sum_{i \in \odot_{j}} \beta_{j, i}^{\prime \eta, \alpha} G_{i, m}^{\alpha, v} u_{j}^{\eta}=\sum_{j \in \Omega^{\prime}} L^{\prime}\left[G_{m}^{\eta, v}\right]_{j} u_{j}^{\eta}
\end{aligned}
$$


Restrict function $G_{j, m}^{\alpha, v}$ to the mesh $\Omega^{\prime}$. Formally, the restriction is a different function, but we denote it below with the same symbol $G_{j, m}^{\alpha, v}$. Apply operator $L^{\prime}$ to the restriction $G_{j, m}^{\alpha, v}$. Obviously, the Eq. (8) will not be valid for operator $L^{\prime}$, since the operators $L^{\prime}$ and $L$ are different on $\partial \Omega^{\prime}$. Instead, for $m \in \Omega^{\prime}$, define the matrix/tensor $b_{j, m}^{v, \eta}$ by the following relation:

$$
L^{\prime}\left[G_{m}^{v, \eta}\right]_{j}=\delta_{j, m} \delta_{v, \eta}+b_{j, m}^{v, \eta}
$$

these values play the role of boundary residue of operator $L^{\prime}$. The values $b_{j, m}^{v, \eta}$ are not equal to 0 only if $j \in \partial \Omega^{\prime}$.

The following "boundary integral representation" for the field can be derived if Eq. (14) is used in Eq. (13):

$$
\sum_{j \in \Omega^{\prime}} f_{j}^{\alpha} G_{j, m}^{\alpha, v}=\sum_{j \in \Omega^{\prime}}\left(\delta_{j, m} \delta_{\eta, v}+b_{j, m}^{\eta, v}\right) u_{j}^{\eta}
$$

And reorganizing terms and deleting those that are null we have the discrete version of the boundary integral equation

$$
u_{m}^{v}=\sum_{j \in \Omega^{\prime}}\left(G_{j, m}^{\alpha, v} f_{j}^{\alpha}-b_{j, m}^{\eta, v} u_{j}^{\eta}\right)=\sum_{j \in \Omega^{\prime}} G_{j, m}^{\alpha, v} f_{j}^{\alpha}-\sum_{j \in \partial \Omega^{\prime}} b_{j, m}^{\eta, v} u_{j}^{\eta} \quad \forall m \in \Omega^{\prime}, v=x, y
$$

A clear paralelism can be done between Eq. (16) and the boundary integral equation used in the BEM applied to the elastodynamics problem (which is a continuous integral equation, see for example [2])

$$
C^{v, \eta} u^{v}\left(\boldsymbol{x}_{m}\right)=\int_{\Gamma} G^{\alpha, v}\left(\boldsymbol{x}_{j}, \boldsymbol{x}_{m}\right) t^{\alpha}\left(\boldsymbol{x}_{j}\right) d \Gamma\left(\boldsymbol{x}_{j}\right)-\int_{\Gamma} T^{\eta, v}\left(\boldsymbol{x}_{j}, \boldsymbol{x}_{m}\right) u^{\eta}\left(\boldsymbol{x}_{j}\right) d \Gamma\left(\boldsymbol{x}_{j}\right)
$$

where $G^{\alpha, v}\left(\boldsymbol{x}_{j}, \boldsymbol{x}_{m}\right)$ is the continuous tensor Green's function (displacement at $\boldsymbol{x}_{j}$ due to unit forces applied at $\boldsymbol{x}_{m}$ ), $C^{v, \eta}$ is the geometry coefficient (it takes different value depending on the smoothness or the angle of the boundary), $u^{v}\left(\boldsymbol{x}_{m}\right)$ is the component $v$ of the displacement at $\boldsymbol{x}_{m}, T^{\eta, v}\left(\boldsymbol{x}_{j}, \boldsymbol{x}_{m}\right)$ is is the surface traction at $\boldsymbol{x}_{j}$ due to unit body force, $t^{\alpha}\left(\boldsymbol{x}_{j}\right)$ is the component $\alpha$ of the surface traction at $\boldsymbol{x}_{j}$ and $\Gamma$ is the boundary of the elastic domain (continuous equivalent to $\partial \Omega^{\prime}$ ). The analogy is clear term by term, with the difference that Eq. (16) is obtained after the discrete formulation of the problem in a grid. It is the final output of the process and it directly leads to a linear system of equations. On the contrary, Eq. (17) is at the starting of the numerical procedure (BEM) and still requires to be discretised (including the numerical integrals). Note that if the nodal forces $f_{j}^{\alpha}$ in Eq. (16) are non-null only on the boundary $\partial \Omega^{\prime}$, the equivalence is as defined. However, if $f_{j}^{\alpha}$ represents also body forces, it can be non-null in other nodes of $\Omega^{\prime} \notin \partial \Omega^{\prime}$. In that case, for the equivalence between Eq. (16) and Eq. (17) to be complete, the integration of body forces in the domain must also be considered by adding

$$
\int_{\Omega^{\prime}} G^{\alpha, v}\left(\boldsymbol{x}_{j}, \boldsymbol{x}_{m}\right) g^{\alpha}\left(\boldsymbol{x}_{j}\right) d \Omega^{\prime}\left(\boldsymbol{x}_{j}\right)
$$

to the right-hand-side of Eq. (17). Where $g$ is a vector of body forces and $\Omega^{\prime}$ accounts now for the equivalent continuous space. 


\subsection{Derivation of discrete analogue of the combined field in- tegral equations}

Returning to the discrete equation Eq. (16), we are particularly interested in the case $m \in \partial \Omega^{\prime}$ to restrict the equation to the boundary. The values of $u_{j}, j \in \partial \Omega^{\prime}$ are the unknowns of this system.

It is also possible to obtain a discrete version of the combined field integral equations (CFIE). This technique enables one to damp the fictitious eigenfrequencies of the exterior problem and also obtain a boundary integral formulation with better regularity properties (see [6] for the scalar Helmholtz equation case). The derivative of the original equation is considered in order to obtain an alternative BIE. These two equations are then combined with a complex coefficient.

Here we apply operator $L^{\prime}$ to both sides of Eq. (16) (this is the discrete analogue of the normal derivative) and take into account that $f_{m}^{\tau}=L^{\prime}[u]_{m}^{\tau}$ :

$$
f_{m}^{\tau}=\sum_{j \in \Omega^{\prime}} \sum_{q \in \Omega^{\prime}} \beta_{m, q}^{\prime \tau, v} G_{j, q}^{\alpha, v} f_{j}^{\alpha}-\sum_{j \in \partial \Omega^{\prime}} \sum_{q \in \Omega^{\prime}} \beta_{m, q}^{\prime \tau, v} b_{j, q}^{\eta, v} u_{j}^{\eta} \quad \forall m \in \Omega^{\prime}
$$

This equation is valid for any $m \in \Omega^{\prime}$, but we are interested only in $m \in \partial \Omega^{\prime}$. The main idea of the CFIE method [6] is to make a linear combination of two integral equations: the original one and its derivative. The uniqueness of the solution is guaranteed through a proper election of the combination constant (in general a complex scalar). Here, Eq. (16) plays the role of the original integral equation while Eq. (19) represents its normal derivative. Thus the CFIE is

$$
\begin{aligned}
u_{m}^{\tau}+\zeta f_{m}^{\tau}= & \sum_{j \in \Omega^{\prime}} G_{j, m}^{\alpha, \tau} f_{j}^{\alpha}-\sum_{j \in \partial \Omega^{\prime}} b_{j, m}^{\eta, \tau} u_{j}^{\eta}+ \\
& \zeta\left(\sum_{j \in \Omega^{\prime}} \sum_{q \in \Omega^{\prime}} \beta_{m, q}^{\prime \tau, v} G_{j, q}^{\alpha, v} f_{j}^{\alpha}-\sum_{j \in \partial \Omega^{\prime}} \sum_{q \in \Omega^{\prime}} \beta_{m, q}^{\prime \tau, v} b_{j, q}^{\eta, v} u_{j}^{\eta}\right) \quad \forall m \in \Omega^{\prime}
\end{aligned}
$$

Eq. (20) is then the discrete equivalent of the CFIE for the elastodynamic problem. $\zeta$ is the combination parameter. The non-singularity of the discrete version of the CFIE equation for the scalar Helmholtz equation for any value of $\zeta$ with non-null imaginary part was proven in [35]. The optimal choice of $\zeta$ for the scalar Helmholtz equation solved by means of BEM can be found in [27].

\subsection{Discrete tensor Green's function}

A method to compute the tensor Green's function is provided here. We assume that $\beta_{j, q}^{\alpha, \eta}$ is given by Appendix A and construct $G_{j, m}^{\alpha, v}$ using the Fourier transform. Let the source node $m$ coincide with the origin $m=0$ (all other cases can be obtained by translating the Green's function along the coordinate lines). Introduce the values $j_{x}$ and $j_{y}$ as integer coordinates of node $j$. Represent the Green's function in the form

$$
G_{j, 0}^{v, \eta}=\frac{1}{(2 \pi)^{2}} \iint_{-\pi}^{\pi} A^{v, \eta}\left(\xi_{1}, \xi_{2}\right) \exp \left\{\mathrm{i}\left(\xi_{1} j_{x}+\xi_{2} j_{y}\right)\right\} d \xi_{1} d \xi_{2}
$$


The inverse transform is as follows:

$$
A^{v, \eta}\left(\xi_{1}, \xi_{2}\right)=\sum_{j_{x}=-\infty}^{\infty} \sum_{j_{y}=-\infty}^{\infty} G_{j, 0}^{v, \eta} \exp \left\{-\mathrm{i}\left(\xi_{1} j_{x}+\xi_{2} j_{y}\right)\right\}
$$

Apply operator $L$ to Eq. (21):

$$
\delta_{\alpha, \eta} \delta_{j, 0}=\frac{1}{(2 \pi)^{2}} \iint_{-\pi}^{\pi} \sigma^{\alpha, v}\left(\xi_{1}, \xi_{2}, k\right) A^{v, \eta}\left(\xi_{1}, \xi_{2}\right) \exp \left\{\mathrm{i}\left(\xi_{1} j_{x}+\xi_{2} j_{y}\right)\right\} d \xi_{1} d \xi_{2}
$$

Noticing that for $j=0 \quad\left(j_{x}=0, j_{y}=0\right)$, we can conclude that $A^{v, \eta}$ must be equal to $\left(\sigma^{-1}\right)^{v, \eta}$. According to the $L$ definition in Eq. (3) based on the linear FEM operator of Appendix A we have

$$
\sigma\left(\xi_{1}, \xi_{2}\right)=\left[\begin{array}{ll}
\sigma^{1,1} & \sigma^{1,2} \\
\sigma^{2,1} & \sigma^{2,2}
\end{array}\right] \quad \sigma^{-1}\left(\xi_{1}, \xi_{2}\right)=\frac{1}{\mathcal{D}}\left[\begin{array}{cc}
\sigma^{2,2} & -\sigma^{2,1} \\
-\sigma^{1,2} & \sigma^{1,1}
\end{array}\right]
$$

with the determinant of $\sigma$

$$
\mathcal{D}=\sigma^{1,1} \sigma^{2,2}-\sigma^{1,2} \sigma^{2,1}
$$

and

$$
\begin{aligned}
\sigma^{1,1} & =-\frac{2 \lambda}{3}\left(1-C_{1}\right)\left(2+C_{2}\right)-\mu\left(4-2 C_{1}-2 C_{1} C_{2}\right)+\rho \omega h^{2} \\
\sigma^{2,2} & =-\frac{2 \lambda}{3}\left(1-C_{2}\right)\left(2+C_{1}\right)-\mu\left(4-2 C_{2}-2 C_{1} C_{2}\right)+\rho \omega h^{2} \\
\sigma^{1,2} \equiv \sigma^{2,1} & =(\lambda+\mu)^{2}\left(1-C_{1}^{2}\right)\left(1-C_{2}^{2}\right)
\end{aligned}
$$

where the following notation is used

$$
C_{1}=\cos \left(\xi_{1}\right) \quad C_{2}=\cos \left(\xi_{2}\right)
$$

Analytical integration with respect to $\xi_{2}$ is performed in Eq. (21). One can note that the integral from $\xi_{2}=-\pi$ to $\xi_{2}=-\pi$ can be understood as an integral over a closed curve in the complex plane, see Fig. 2(a). Here it can be seen that the integrals on $\Gamma_{-\pi}$ and $\Gamma_{+\pi}$ cancel each other. The integral along $\Gamma_{\infty}$ is null due to exponential decay of the function. Thus,

$$
\oint=\int_{-\pi}^{\pi}+\int_{\Gamma_{\pi}}+\int_{\Gamma_{\infty}}+\int_{\Gamma_{-\pi}}=\int_{-\pi}^{\pi}
$$

Using Cauchy's theorem, obtain

$$
\begin{aligned}
{\left[\begin{array}{ll}
G^{x, x} & G^{x, y} \\
G^{y, x} & G^{y, y}
\end{array}\right]_{j, 0}=\frac{2 \pi \mathrm{i}}{(2 \pi)^{2}} \int_{-\pi}^{\pi}\left[\begin{array}{cc}
\sigma^{2,2} & -\sigma^{2,1} \\
-\sigma^{1,2} & \sigma^{1,1}
\end{array}\right] \frac{\exp \left\{\mathrm{i}\left(\xi_{1} j_{x}+j_{y} \Xi_{1}\left(\xi_{1}\right)\right)\right.}{\left.\left.\frac{\partial \mathcal{D}}{\partial C_{2}}\right|_{C_{2}^{* 1}} \frac{\partial C_{2}}{\partial \xi_{2}}\right|_{\xi_{2}=\Xi_{1}\left(\xi_{1}\right)}} d \xi_{1}+} \\
+\frac{2 \pi \mathrm{i}}{(2 \pi)^{2}} \int_{-\pi}^{\pi}\left[\begin{array}{cc}
\sigma^{2,2} & -\sigma^{2,1} \\
-\sigma^{1,2} & \sigma^{1,1}
\end{array}\right] \frac{\exp \left\{\mathrm{i}\left(\xi_{1} j_{x}+j_{y} \Xi_{2}\left(\xi_{1}\right)\right)\right.}{\left.\left.\frac{\partial \mathcal{D}}{\partial C_{2}}\right|_{C_{2}^{* 2}} \frac{\partial C_{2}}{\partial \xi_{2}}\right|_{\xi_{2}=\Xi_{2}\left(\xi_{1}\right)}} d \xi_{1}
\end{aligned}
$$




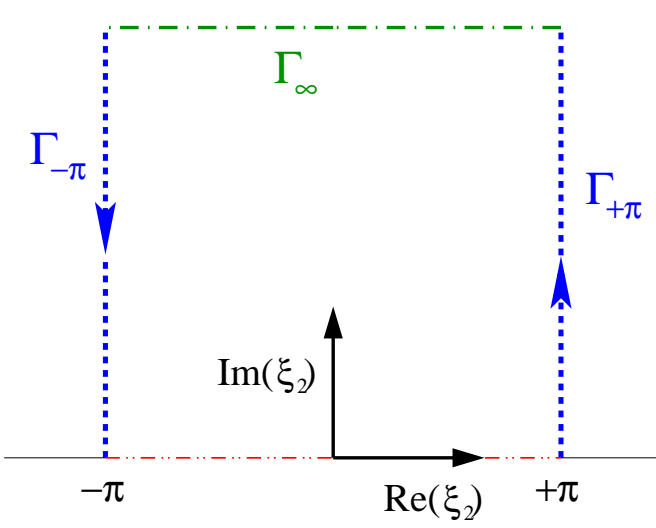

(a)

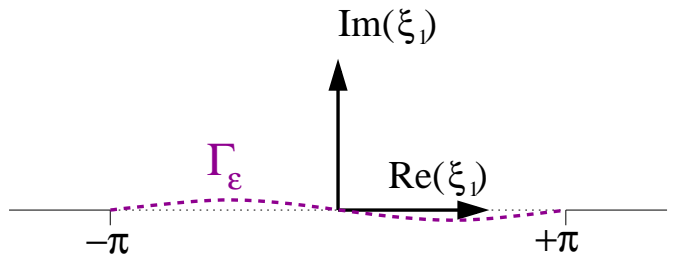

(b)

Figure 2: Integration paths in the complex plane: (a) Closed path to integrate on the variable $\xi_{2}$; (b) Detail of the path $\Gamma_{\epsilon}$ used to make the numerical integral on $\xi_{1}$.

where $\Xi_{1}\left(\xi_{1}\right)$ and $\Xi_{2}\left(\xi_{1}\right)$ are the roots of quadratic equation $\mathcal{D}\left(\xi_{2}\right)=0, C_{2}^{* 1}=$ $\cos \left(\Xi_{1}\left(\xi_{1}\right)\right)$ and $C_{2}^{* 2}=\cos \left(\Xi_{2}\left(\xi_{1}\right)\right)$.

Finally the integral in Eq. (30) is evaluated numerically. The integration path in the complex plane $\xi_{1}$, see Fig. 2(b) is chosen in order to avoid the singularities and to include into the upper half plane only the poles only corresponding to outgoing waves. The curve $\Gamma_{\varepsilon}$ is in practise defined as a slight perturbation of the segment $[-\pi, \pi]$ of the real axis by means of a sine function.

The integral representation described above is valid for a point with $j_{x} \geq 0$ and $j_{y} \geq 0$. The discrete Green's function must be symmetrized to cover all possible nodes of the grid. The sense and direction of the displacements in each quadrant is shown in Fig. 3. The symmetrisation is performed as follows:

$$
\begin{array}{r}
G_{\left(-j_{x}, j_{y}\right),(0,0)}^{v, \eta}=G_{\left(j_{x},-j_{y}\right),(0,0)}^{v, \eta}=(-1)^{v+\eta} G_{\left(j_{x}, j_{y}\right),(0,0)}^{v, \eta} \\
G_{\left(-j_{x},-j_{y}\right),(0,0)}^{v, \eta}=G_{\left(j_{x}, j_{y}\right),(0,0)}^{v,}
\end{array}
$$
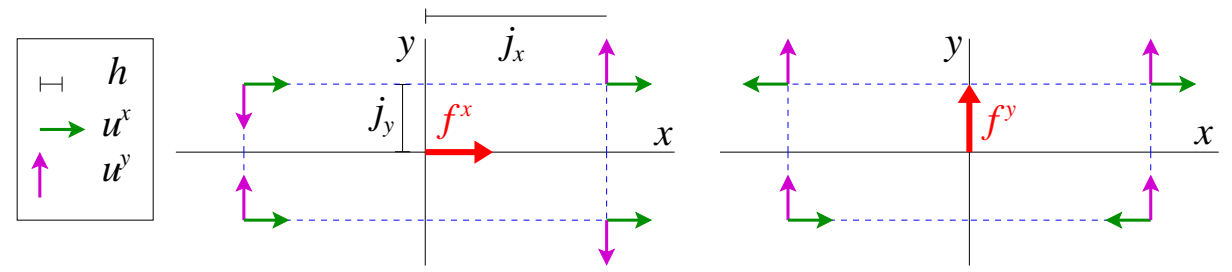

Figure 3: Sketch of the displacements in an arbitrary point of each quadrant caused by a point force applied at $(0,0)$. 


\subsection{Implementation details}

\subsubsection{Boundary conditions}

Equation (7) can describe a problem with known traction or known displacement on the boundary (essential or natural boundary conditions, respectively). In both cases the coefficients $\beta^{\prime}$ are symmetrical. For the numerical examples shown here, Lagrange multipliers have been used in order to block the boundary displacements [51]. If there is a continuous field of uniformly distributed forces per unit volume, they can be taken into account as described in Appendix A.

Another quite usual situation in elastodynamics is the diffraction problem, i.e. there is an incident field and, say, a traction-free scatterer in the medium. It is necessary to find the scattered field. Homogenous equation (7) should be valid for all nodes:

$$
L^{\prime}\left[u^{\text {total }}\right]_{j}^{\alpha}=L^{\prime}\left[u^{\text {incident }}+u^{\text {scattered }}\right]_{j}^{\alpha}=0
$$

When solving for the scattered field only, the force vector has form

$$
f_{j}^{\alpha}=-L^{\prime}\left[u^{\text {incident }}\right]_{j}^{\alpha} .
$$

\subsubsection{Coupling of the BAE formulation with FEM}

As described in [36], an important drawback of BAE formulations is the error produced when geometries that do not fit in the grid shape (here square) must be represented. This can be overcome by placing a thin layer of finite elements (not necessarily regular squares, see Fig. 4) between the object boundary and the closest grid-shaped representation. The coupling of the boundary algebraic formulation with a thin layer of finite elements which was more formally explained in [34] for the Helmholtz equation, is briefly summarised here below for the case of elastodynamic scattering.

The coupling between FEM and BAE is done here in a simple way by considering matching finite element mesh and BAE nodes. The only purpose is to show that when an improvement on the boundary description is required, the coupling with a technique that allows a better description of the geometry can reduce the geometry error and improve the results.

The domain discretised by means of triangular finite elements surrounding the scatterer is denoted by $\Omega_{\text {int }}$ while $\Omega_{\text {ext }}$ is the external grid where BAE formulation is considered (it is equivalent to $\Omega^{\prime}$ ). The internal boundary that defines the scatterer is $\Gamma_{\text {int }}$. Finite elements can in general approximate better this geometry. In particular, if it is not grid-shaped.

The interface between FEM and BAE domains is denoted by $\Gamma_{\text {ext }}$. A FEM mesh that matches the grid is used. It means that all the nodes (the vertices of the triangles and the nodes of the grid) are coincident on $\Gamma_{\text {ext }}$.

A projector tensor $\Pi_{m, n}^{\eta, v}$ is defined in order to identify the nodes in $\Omega_{\text {int }}$ that belong to $\Gamma_{\text {ext }}$. Here $n$ are the nodes of the FEM mesh (all the nodes $N_{\text {int }}$ used to discretise $\Omega_{\text {int }}$ ). And $m$ are those nodes in $\Gamma_{\text {ext }}$ (all the $N_{\text {ext }}$ nodes in $\Gamma_{\text {ext }}$ ). The $m$ nodes are double in the sense that belong to the FEM part of the problem but are also affected by the BAE formulation.

$$
\Pi_{m, n}^{\eta, v}=\pi_{m, n} \delta_{\eta, v} \quad \eta, v=x, y \quad n=1, \ldots, N_{\text {int }} \quad m=1, \ldots, N_{\text {ext }}
$$




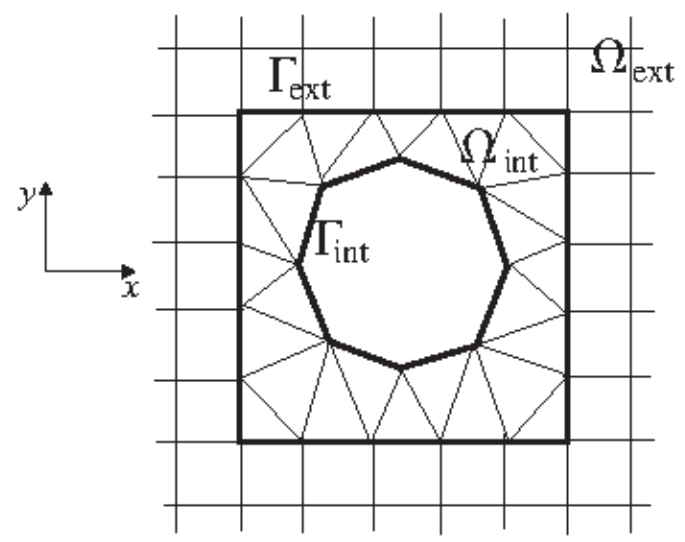

Figure 4: Coupling of an internal domain discretised by means of linear FEM triangles and an external grid where BAE is considered.

and

$$
\pi_{m, n}= \begin{cases}1, & m=n, \text { coincident nodes } \\ 0, & \text { otherwise }\end{cases}
$$

and $\pi_{m, n}^{T}=\pi_{n, m}$.

The continuity of the nodal displacement in the BAE-FEM interface is imposed

$$
u_{\text {int }}^{\eta}=u_{\text {ext }}^{\eta} \quad x \in \Gamma_{\text {ext }} \quad \eta=x, y
$$

This can be expressed as

$$
\left(u_{\text {ext }}\right)_{m}^{\eta}=\Pi_{m, n}^{\eta, v}\left(u_{\text {int }}\right)_{n}^{v}
$$

In addition, the internal forces (between $\Omega_{\text {int }}$ and $\Omega_{\text {ext }}$ ) must be balanced. They are represented here by a fictitious nodal force vector $\mathbf{g}_{\text {ext }}$ at the nodes in the interface $\Gamma_{\text {ext }}$. This is only required as a formulation tool in order to be able to consider the BAE and FEM domains isolated. It represents the forces acting on the boundary $\Gamma_{\text {ext }}$ of $\Omega_{\text {ext }}$ due to the truncation of the grid. These forces allow to impose the continuity of the displacement field as defined in Eq. (37).

If we consider the domain $\Omega_{\text {ext }}$ (which plays the role as $\Omega^{\prime}$ ), a BAE formulation affecting the nodes on $\Gamma_{\text {ext }}$ (which plays the role as $\partial \Omega^{\prime}$ ) can be derived as before with the only difference that now there exist interaction forces represented by $\mathbf{g}_{\text {ext }}$. They are a priori unknown and play the role of $f_{j}^{\alpha}$ in Eq. (13). They can be understood as the forces from the FEM part to the BAE boundary or the forces on $\Omega_{\text {ext }}$ due to its isolation from $\Omega_{\text {int }}$. Its algebraic representation is done by means of a 'coupling' matrix C. It can be obtained from the terms containing nodal forces in the righthand-side of Eq. (16) or Eq. (20) (with CFIE). A $2 \times 2$ box of $\mathbf{C}$ that relates the displacements at the $m$ BAE node with the interaction forces at the node $j$ belonging to the FEM mesh is

$$
C_{m, j}^{\alpha, v}=G_{j, m}^{\alpha, v}+\zeta\left(\sum_{q \in \Omega^{\prime}} \beta_{m, q}^{v, \eta} G_{j, q}^{\alpha, \eta}-1\right), \quad \alpha, v=x, y
$$


A ext is the $N_{\text {ext }} \times N_{\text {ext }}$ matrix form of Eq. (20), by taking into account all the terms without nodal force (already included in $\mathbf{C}$ )

$$
A_{m, j}^{\alpha, v}=\delta^{\alpha, v}+b_{j, m}^{\alpha, v}+\zeta \sum_{q \in \Omega^{\prime}} \beta_{m, q}^{\alpha, \tau} b_{j, q}^{v, \tau} \quad \forall m, j \in \Omega^{\prime}, \quad \alpha, v=x, y
$$

These allow the matrix formulation of the BAE part of the problem.

Considering the domain $\Omega_{\text {int }}$ which is described by means of finite elements we have the following equilibrium equation in matrix form

$$
\mathbf{A}_{\text {int }} \mathbf{u}_{\text {int }}=\mathbf{f}_{\text {int }}-\boldsymbol{\Pi}^{T} \mathbf{g}_{\text {ext }}
$$

where $\mathbf{A}_{\text {int }}$ is the $N_{\text {int }} \times N_{\text {int }}$ dynamic FEM matrix based on the linear finite element theory for plane strain [24, 51]. The boundary conditions described in Section 2.6.1 are considered, leading to a vector of nodal forces $\mathbf{f}_{\text {int }}$ (it is in general non-null only in the nodes that belong to $\Gamma_{\text {int }}$ ). These two aspects are common of the usual FEM formulations. $-\boldsymbol{\Pi}^{T} \mathbf{g}_{\text {ext }}$ represents the interaction forces acting on the boundary $\Gamma_{\text {ext }}$ when $\Omega_{\text {int }}$ is considered as an isolated domain.

The matrix form of the whole problem including the FEM part close to the scatterer and the BAE part in order to truncate the domain is

$$
\left[\begin{array}{ccc}
\mathbf{A}_{\text {ext }} & \mathbf{0} & -\mathbf{C} \\
\mathbf{0} & \mathbf{A}_{\text {int }} & \boldsymbol{\Pi}^{T} \\
\mathbf{I} & -\boldsymbol{\Pi} & \mathbf{0}
\end{array}\right]\left[\begin{array}{l}
\mathbf{u}_{\text {ext }} \\
\mathbf{u}_{\text {int }} \\
\mathbf{g}_{\text {ext }}
\end{array}\right]=\left[\begin{array}{c}
\mathbf{0} \\
\mathbf{f}_{\text {int }} \\
\mathbf{0}
\end{array}\right]
$$

The first block-row in Eq. (42) represents the BAE part of the problem. The second, the FEM part. And finally the third block-row represents the continuity of nodal displacements in Eq. (37) expressed in matrix form by means of Eq. (35).

\subsubsection{Extension to $3 \mathrm{D}$ or other elasticity formulations}

As mentioned before, the BAE formulation in Sections 2.2, 2.3 and 2.4 could be applied to three-dimensional or other elastodynamics problems. The ingredients for the method are three: $i$ ) to be able to generate a free field operator; ii) to provide physical meaning to the operator truncation; iii) to compute a discrete Green's function for the chosen geometry (in the case presented here, rectangular grids).

The first one can be achieved without problems because FEM formulations and element technology for many elastodynamics problems (i.e. three-dimensional or axisymmetry) are available. An equivalent to Appendix A can be obtained by the assembly of a patch of elements around a node. The truncation of this element patch has, in general, physical meaning and the second of the mentioned ingredients is satisfied. This is especially true if the elements considered are linear. The third one, obtaining a discrete Green's function (equivalent to Section 2.5), is the step that can suppose some difficulty.

Some aspects related with computational efficiency are more or less similar to other boundary techniques. In particular, it is probable that an equivalent to fast 
multipole technique [7] would be needed in order to deal with large three-dimensional domains which imply large loops on boundary nodes. It is also probable to improve the efficiency of the BAE method by means of an alternative computation of the discrete Green's function in the far field. For all these, an extension to three dimensions of the method is possible even if it is not straightforward. However, the numerical examples provided in the following sections are based on two-dimensional domains.

\section{$3 \quad$ Numerical examples}

Different aspects of the numerical method are illustrated here by means of some examples. The relative error is calculated by means of the energy norm on the boundary. If the displacement field has non-null components in all the directions

$$
\begin{aligned}
& e=\sqrt{\frac{\int_{\partial \Omega^{\prime}}\left|\left(u^{x}-u_{\mathrm{ref}}^{x}\right)\right|^{2}+\left|\left(u^{y}-u_{\mathrm{ref}}^{y}\right)\right|^{2} d S}{\int_{\partial \Omega^{\prime}}\left|u_{\mathrm{ref}}^{x}\right|^{2}+\left|u_{\mathrm{ref}}^{y}\right|^{2} d S}} \\
& \simeq \sqrt{\frac{\sum_{i \in \Gamma_{\mathrm{ext}}}^{n}\left|\left(u_{i}^{x}-u_{i, \mathrm{ref}}^{x}\right)\right|^{2}+\left|\left(u_{i}^{y}-u_{i, \mathrm{ref}}^{y}\right)\right|^{2}}{\sum_{i \in \Gamma_{\mathrm{ext}}}^{n}\left|u_{i, \mathrm{ref}}^{x}\right|^{2}+\left|u_{i, \mathrm{ref}}^{y}\right|^{2}}}
\end{aligned}
$$

and if it has only radial component

$$
e=\sqrt{\frac{\int_{\partial \Omega^{\prime}}\left|u^{r}-u_{\mathrm{ref}}^{r}\right|^{2} d S}{\int_{\partial \Omega^{\prime}}\left|u_{\mathrm{ref}}^{r}\right|^{2} d S}} \simeq \sqrt{\frac{\sum_{i \in \Gamma_{\mathrm{ext}}}^{n}\left|\left(u_{i}^{r}-u_{i, \mathrm{ref}}^{r}\right)\right|^{2}}{\sum_{i \in \Gamma_{\mathrm{ext}}}^{n}\left|u_{i, \mathrm{ref}}^{r}\right|^{2}}}
$$

where $\partial \Omega^{\prime}$ can represent the boundary of a solid (interior problems) or the boundary of a scatterer, typically the boundary of a hole inside an unbounded elastic medium (exterior problems). The subscript 'ref' denotes the reference solution. The examples shown here have exact solution with available analytical expression. This is taken as reference. The error is computed by means of the discrete version of the norms placed in the righ-hand-side of Eqs. (43) and (44). The material properties of the isotropic elastic medium considered in these examples are those of Table 1.

Table 1: Mechanical properties of the linear elastic medium.

\begin{tabular}{lll} 
Meaning & Symbol & Value \\
\hline Young module & $E$ & $3.0 \cdot 10^{10} \mathrm{~N} / \mathrm{m}^{2}$ \\
Poisson ratio & $\nu$ & 0.2 \\
Density & $\rho$ & $2200 / \mathrm{kg} / \mathrm{m}^{3}$
\end{tabular}

\subsection{Exterior problem: cylindrical cavity subjected to pulsat- ing pressure}

The first example is a cylindrical cavity in an unbounded elastic medium subjected to pulsating internal pressure. The pressure acts in the radial direction and it is 
a uniform distribution. The main advantage of considering this problem is that a straightforward analytical solution is available [12, 21]

$$
u^{r}(r)=\frac{R}{\mu} \frac{P H_{1}^{(1)}\left(\Omega_{P}\right)}{2 H_{1}^{(1)}\left(\Omega_{0}\right)-a^{-2} \Omega_{0} H_{0}^{(1)}\left(\Omega_{0}\right)}, \quad r \geq R
$$

and

$$
a=\frac{v_{S}}{v_{P}} \quad \Omega_{P}=\frac{\omega r}{v_{P}} \quad \Omega_{0}=\frac{\omega R}{v_{P}}
$$

with $R$ the radius of the cylindrical cavity, $P$ the uniform pulsating pressure, $v_{S}=$ $\sqrt{\mu / \rho}$ the S-wave velocity, $v_{P}=v_{S} \sqrt{2(1-\nu) / 1-2 \nu}$ the P-wave velocity, $\nu$ the Poisson's ratio, $H_{n}^{(1)}$ the first Hankel function of order $n$ (Bessel function of third kind), $\Omega_{P}$ the dimensionless frequency for the $\mathrm{P}$-wave or compressional wave, see for example [31]. This exact solution is evaluated at the nodes describing the scatterer. They can be grid nodes (if only BAE is considered) or nodes of the FEM mesh (if FEM is coupled with BAE). The comparison between the numerical solution and the exact solution is done at these nodes. The paremeter $\Omega_{0}$ is used as dimensionless frequency in the results shown below.

The main drawback of the BAE method is that the curved geometry must be approximated by means of a rectangular grid-shaped contour. In that situation the BAE method always has some numerical error caused by the geometry approximation. Figs. 5 and 6 show the results for a cavity with circular cross-section and a radius $R=3 \mathrm{~m}$ solved with BAE. A unitary internal pressure $(P=1)$ is considered. The real and imaginary parts of the radial displacement are shown in Fig. 5. The numerical value is the average for all the angles. The general trend of the exact solution is followed by the computations. However, computations with grid sizes $h=1 \mathrm{~m}$ and $h=0.5 \mathrm{~m}$ have considerable errors.

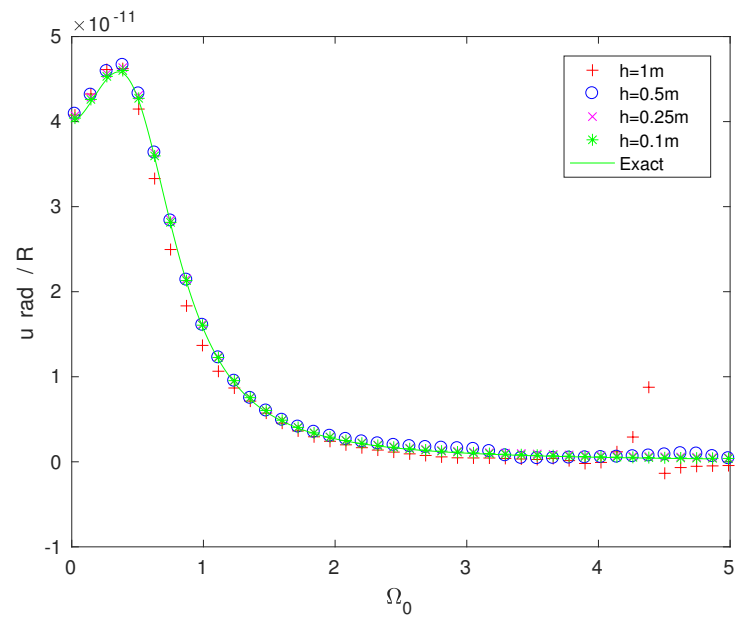

(a) Real

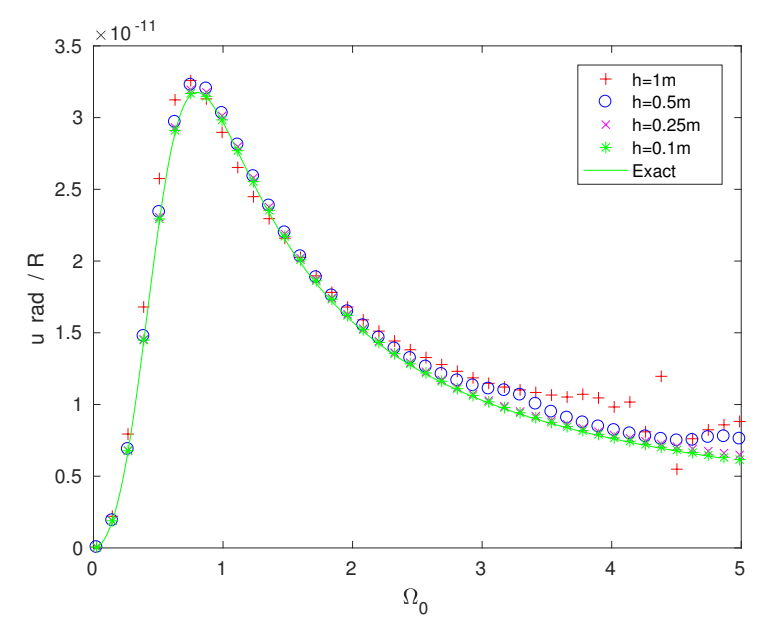

(b) Imag

Figure 5: Cylindrical cavity (with radius $3 \mathrm{~m}$ ) subjected to pulsating pressure inside. The solution only varies in the radial direction (cylindrical symmetry). The numerical solution is obtained with BAE. Plot of the solution, radial displacement (Eq. (45)) : (a) real; (b) imaginary. 
This can be seen more clearly in Fig. 6 where the relative error is plot. Fig. 6(b) shows the dependence of the error with frequency. A global trend for this example is that the error is reduced by 10 each $1000 \mathrm{~Hz}$. Fig. 6(a) shows the variation of the error due to the reduction of the grid size for several frequencies. The slope is quite irregular and depends on the frequency of the problem. For this example, $\log _{10}(e) / \log _{10}(h)$ is around $1 / 2$.

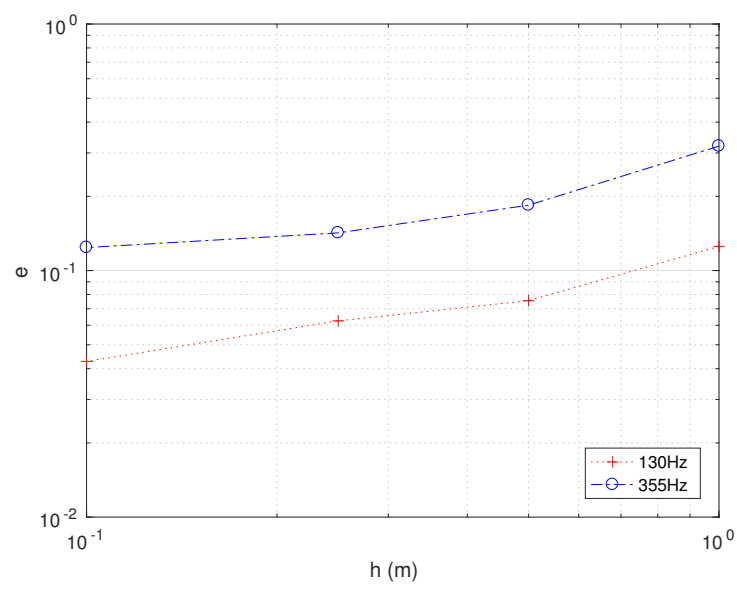

(a)

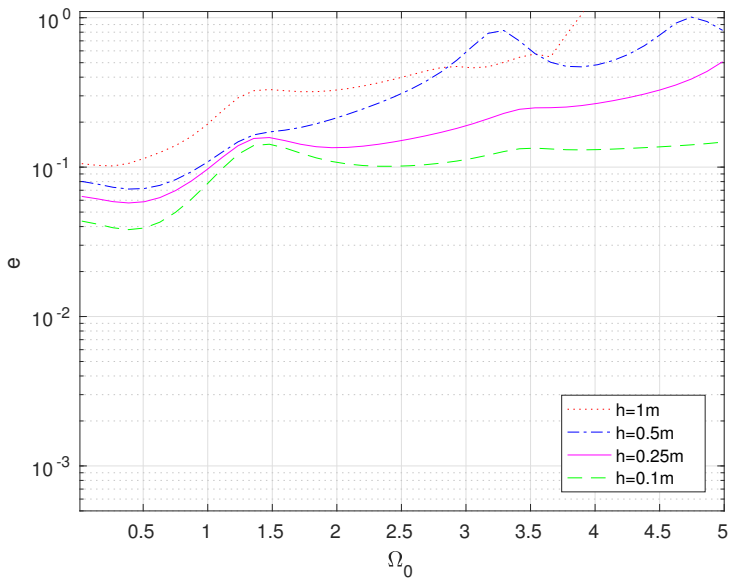

(b)

Figure 6: Cylindrical cavity (with radius $3 \mathrm{~m}$ ) subjected to pulsating pressure inside. The solution only varies in the radial direction (cylindrical symmetry). The numerical solution is obtained with BAE. Difference with respect to the exact solution: (a) Dependence of the relative error on the grid size for several frequencies (frequency $130 \mathrm{~Hz}$ with $\Omega_{0}=0.67$, and frequency $355 \mathrm{~Hz}$ with $\Omega_{0}=1.83$ ); (b) Dependence of the relative error on the problem frequency.

The error of BAE method for problems with curved boundaries is caused mainly by the geometry error. To study better the performance of BAE and avoid this drawback, a thin layer of linear finite elements is considered around the circle([24] is used here). When this is done, the frequency and grid-size dependence of the error can be analysed in more details. The meshes are shown in Fig. 7. They are generated with Gmsh [14]. The element/grid size is modified in the analysis. However, the external boundary that determines the BAE contour remains constant. The element/grid sizes in Fig. 7(b) and (c) allows a thinner FEM layer, but it seems to us adequate not to move the BAE contour in order to make a fair comparison.

Figs. 8 and 9 are the results obtained with the FEM-BAE coupling. In general, a much better agreement with the exact solution can be seen in Fig. 8. The differences are only visible for the coarsest mesh/grid where $h=1 \mathrm{~m}$ and at the highest of the computed frequencies.

The evolution of the error with frequency is plot in Fig. 9. All the curves show a more linear trend. This clearly denotes that most of the variations and oscillations observed in Fig. 6 are caused by the approximation of the circle shape. The error $e$ is reduced by 10 (or a bit more, especially in the case with grid size $h=1 \mathrm{~m}$ ) every $1000 \mathrm{~Hz}$. This global trend is not largely modified by the inclusion of the FEM layer.

However, the error reduction due to the grid/element size reduction is now largely 


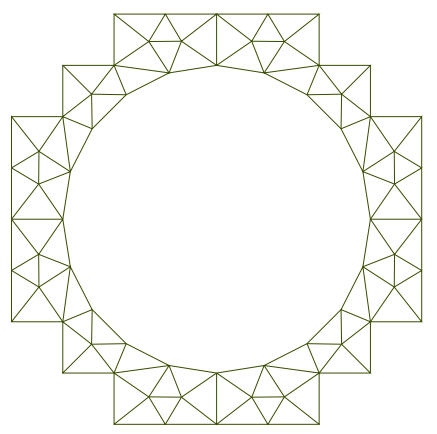

(a)

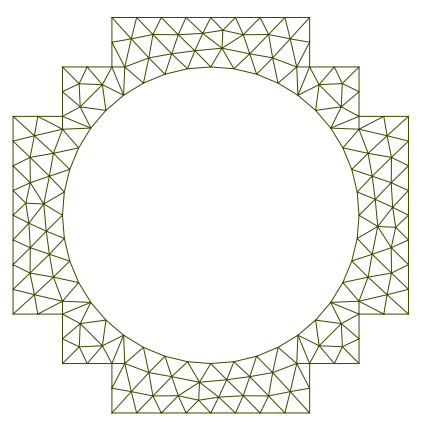

(b)

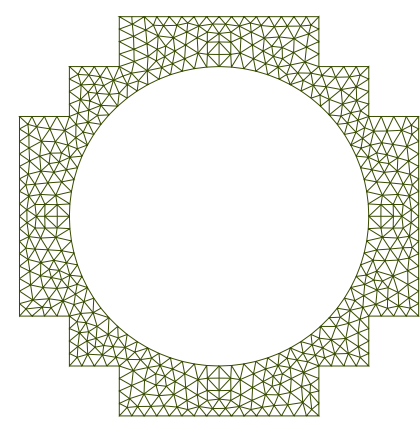

(c)

Figure 7: Thin layer of finite elements around a circular boundary with radius $R=3$ m. Element sizes: (a) $h=1 \mathrm{~m}$; (b) $h=0.5 \mathrm{~m}$; (c) $h=0.25 \mathrm{~m}$.

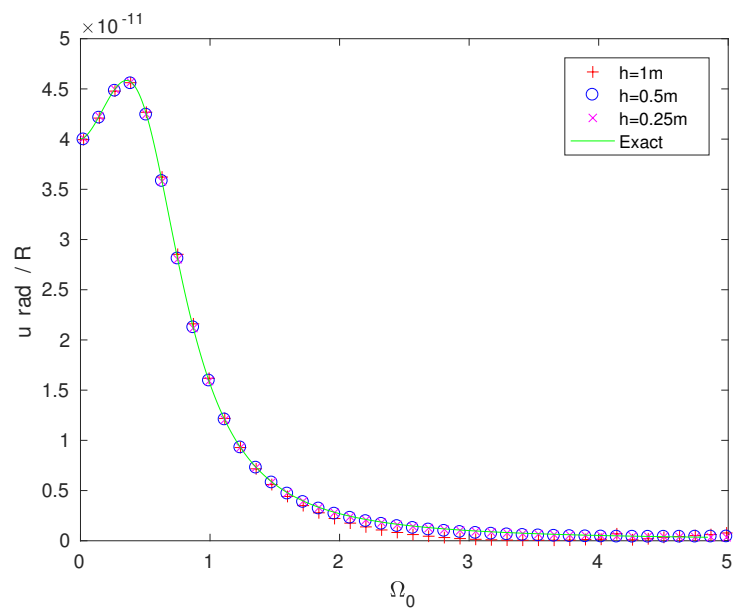

(a) Real

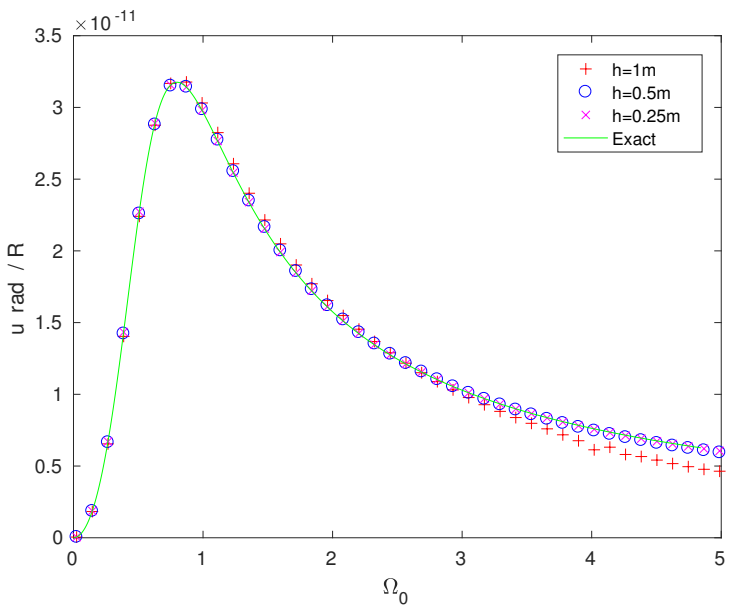

(b) Imag

Figure 8: Cylindrical cavity (with radius $3 \mathrm{~m}$ ) subjected to pulsating pressure inside. The solution only varies in the radial direction (cylindrical symmetry). The numerical solution is obtained with the coupling of FEM and BAE. Plot of the solution, radial displacement (Eq. (45)) : (a) real; (b) imaginary.

improved by the FEM layer, as shown by the lines in Fig. 9(a). Now the slope computed as $\log _{10}(e) / \log _{10}(h)$ is close to 1 .

An equivalent analysis has been performed with a BAE boundary with square shape (see the three meshes in Fig. 10 (a)) instead of a thin layer of finite elements. The results are very similar. So, it indicates that the difference between a squareshaped mesh and a staircased mesh is not meaningful. Taking into account the important reduction of nodes (and consequently size of matrices and computational costs in general), the recommendation is then to use as less finite elements as possible, forming a thin layer around the scatterer.

Finally, the influence of the mesh truncation distance has been checked. Four meshes of Fig. 10 (a) with a constant element/grid size $h=0.25 \mathrm{~m}$ have been considered. The radius of the circle scatterer was constant $(R=3 \mathrm{~m})$ while the size of the 


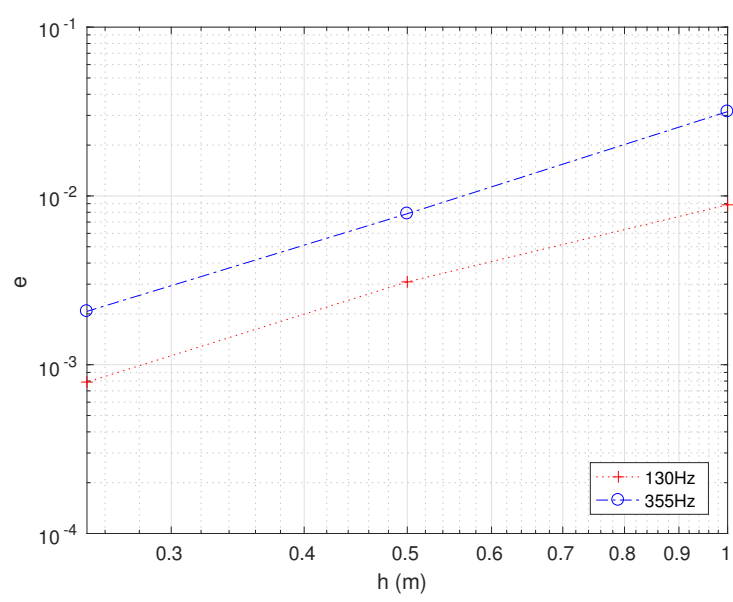

(a)

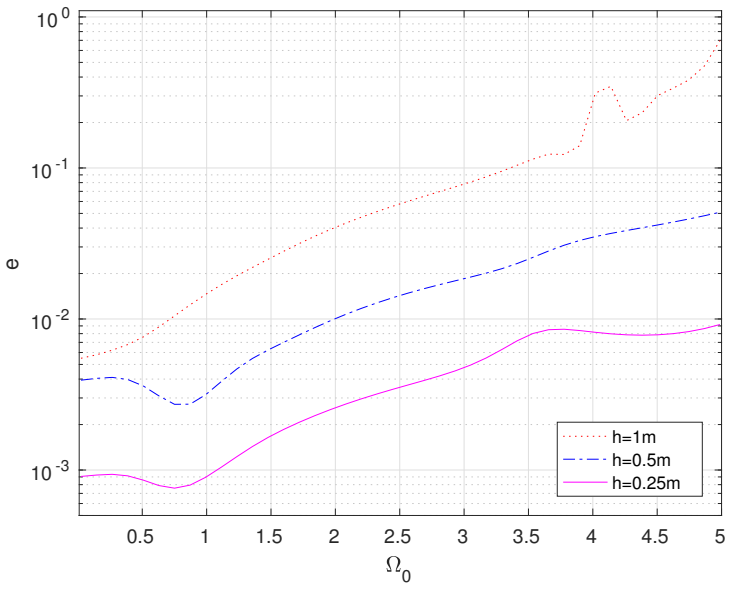

(b)

Figure 9: Cylindrical cavity (with radius $3 \mathrm{~m}$ ) subjected to pulsating pressure inside. The solution only varies in the radial direction (cylindrical symmetry). The numerical solution is obtained with the coupling of FEM and BAE. Difference with respect to the exact solution: (a) Dependence of the relative error on the grid size for several frequencies (frequency $130 \mathrm{~Hz}$ with $\Omega_{0}=0.67$, and frequency $355 \mathrm{~Hz}$ with $\Omega_{0}=1.83$ );

(b) Dependence of the relative error on the problem frequency.

external FEM box has been modified, being the length of the square side $L=8,12,16$ m. The number of nodes in the FEM mesh and the number of grid nodes at the external boundary where the mesh is truncated are listed in Table 2. This is directly related with the computational burden (size of linear systems to solve, computation of matrices in Eq. (42) etc.). It is clear that a truncation of the mesh as close to the scatterer (internal boundary) as possible, largely reduces the number of nodes involved in the problem.

Fig. 10 (b) shows the relative error for any of the considered meshes (truncation distance). In general, it can be seen that the differences between each case are not very large. It suggests that a good strategy is to truncate the FEM mesh as close as possible to the scatterer in order to reduce the number of nodes. The gain in computational burden would be better used to reduce the element and grid sizes which is an aspect that influences more the reduction of numerical error (see, Fig. 9).

Table 2: Number of nodes in the meshes shown in Fig. 10 (a).

\begin{tabular}{l|cccc} 
Meaning & Thin & $L=8 \mathrm{~m}$ & $L=12 \mathrm{~m}$ & $L=16 \mathrm{~m}$ \\
\hline$\sharp$ FEM nodes & 344 & 580 & 2090 & 4058 \\
$\sharp$ BAE nodes & 148 & 128 & 192 & 256
\end{tabular}




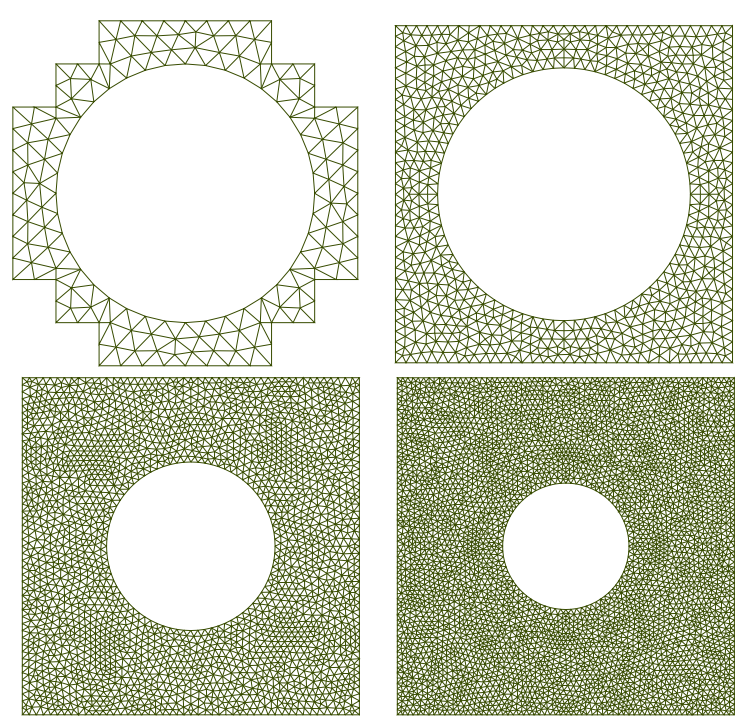

(a)

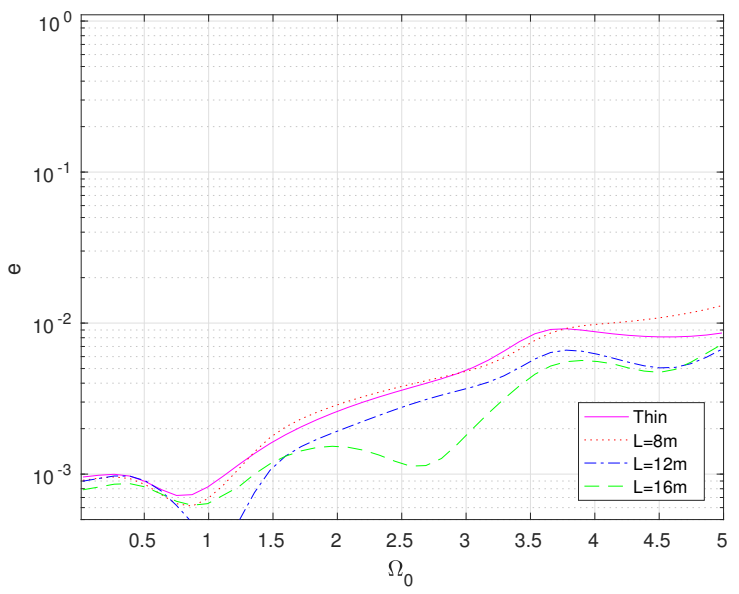

(b)

Figure 10: Cylindrical cavity (with radius $3 \mathrm{~m}$ ) subjected to pulsating pressure inside. The solution only varies in the radial direction (cylindrical symmetry). The numerical solution is obtained with the coupling of FEM and BAE. Difference with respect to the exact solution. Influence of the size of the FEM layer that surrounds the circle (distance at which the mesh is truncated). Constant grid and finite element size $(0.25 \mathrm{~m})$ : (a) the meshes considered: 'thin' layer of finite elements around the circle (double element size for the sake of clarity), and square boxes of size $L=8,12,16 \mathrm{~m}$ (b) Dependence of the relative error on the problem frequency.

\subsection{Effect of CFIE formulation on the matrix condition num- ber}

One of the drawbacks of the numerical methods for exterior problems that are based on a boundary integral formulation is the singularity of the problem at some fictitious eigenfrequencies. These eigenfrequencies have no physical meaning and correspond to the eigenfrequencies of the complementary interior problem. Typically, this complementarity concept is defined in terms of the boundary conditions: free surface with no imposed force on the exterior problem and surface with blocked displacements for the interior problem or vice-versa.

This aspect is probably more important and has been studied better for the scalar Helmholtz equation, for which several techniques were proposed to correct this drawback. In solid mechanics, less attention has been paid probably due to relatively high damping required to model some of the usual materials (especially in geological applications involving the soil). However, there exist several methods to overcome fictitious eigenfrequencies for BEM in elastodynamics [20, 19, 32, 23, 39, 37, 8].

The BAE formulation presented here overcomes this difficulty by means of a discrete version of the combined field equations (CFIE), see Eq. (20). The performance of this solution is demonstrated for an exterior problem: the scattering by a rectangle. It is the same problem as in Section 3.1 but the shape of the hole inside the unbounded elastic domain is now a rectangle instead of a circle. The dimensions of 
the rectangle are $L_{x} \times L_{y}$. Internal oscillating pressure is imposed on each side of the rectangle, with value 1 .

The rectangular shape has been chosen in order to avoid the interference of the geometry approximation in the numerical results (a rectangle boundary is exactly described by the BAE method). Also, for this section the coupling with FEM is not considered in order to avoid some masking effect caused by the FEM part of the matrices and focus the interest only in the BAE method.

In all studied problems no noticeable difference between the results obtained with Eq. (16) and Eq. (20) have been found. Very precise computations are needed in order to see the effect of fictitious eigenfrequencies. Table 3 summarizes the results around one of these eigenfrequencies. It corresponds to the scattering of a rectangleshaped cavity with dimensions $L_{x}=20 \mathrm{~m}$ and $L_{y}=16 \mathrm{~m}$ due to a uniform oscillating pressure. The value of $\zeta$ is $-\mathrm{i}$. Other values of $\zeta=\mathrm{i}, \frac{-\mathrm{i}}{\omega}, \ldots$ have been used with minor or null modification of the results in Table 3. These parameters are chosen because they are the typical ones used in the BEM literature of the Helmholtz equation [27] (what it is clear is that non-null imaginary part of $\zeta$ is needed.).

For a series of frequencies approaching to the value of the fictitious eigenfrequency $119.902663471 \mathrm{~Hz}$, the condition number of the system matrix using Eq. (16) and the condition number of the CFIE system matrix using Eq. (20) are shown. The second one remains almost constant while for the first one a sudden increase of the condition number around the fictitious frequency can be seen. This value of the condition number could tend to infinity as long as the fictitious frequency could be evaluated more accurately.

The right column is a measure of the difference between the standard solution based on Eq. (16) and the CFIE solution based on Eq. (20). They are compared according to Eq. (43). When approaching to the continuous eigenfrequency the difference increases but it is still small. Again with a value accurate enough of the eigenfrequency, this difference should be larger due to numerical errors in the standard solution.

Table 3: Matrix condition number around one of the fictitious frequencies of the problem.

\begin{tabular}{lccc} 
Freq. $\frac{\omega}{2 \pi}=f(\mathrm{~Hz})$ & Cond. Eq. (16) & Cond. CFIE, Eq. $(20)$ & $e_{\text {standard }-C F I E}$ \\
\hline 117.000000000 & $3.51 \cdot 10^{1}$ & $1.53 \cdot 10^{2}$ & $6.6 \cdot 10^{-7}$ \\
118.000000000 & $5.35 \cdot 10^{1}$ & $1.37 \cdot 10^{2}$ & $5.4 \cdot 10^{-7}$ \\
119.000000000 & $1.13 \cdot 10^{2}$ & $1.46 \cdot 10^{2}$ & $1.0 \cdot 10^{-6}$ \\
119.900000000 & $3.83 \cdot 10^{4}$ & $1.76 \cdot 10^{2}$ & $9.0 \cdot 10^{-7}$ \\
119.902200000 & $2.20 \cdot 10^{5}$ & $1.76 \cdot 10^{2}$ & $1.4 \cdot 10^{-6}$ \\
119.902650000 & $7.56 \cdot 10^{6}$ & $1.76 \cdot 10^{2}$ & $1.3 \cdot 10^{-6}$ \\
119.902662000 & $6.83 \cdot 10^{7}$ & $1.76 \cdot 10^{2}$ & $3.3 \cdot 10^{-6}$ \\
119.902663000 & $2.11 \cdot 10^{8}$ & $1.76 \cdot 10^{2}$ & $3.3 \cdot 10^{-6}$ \\
119.902663450 & $4.39 \cdot 10^{9}$ & $1.76 \cdot 10^{2}$ & $1.2 \cdot 10^{-2}$ \\
119.902663471 & $1.52 \cdot 10^{10}$ & $1.76 \cdot 10^{2}$ & $2.9 \cdot 10^{-2}$
\end{tabular}


It is clear that the frequency band where the fictitious eigenfrequencies have some effect on the solution is extremely narrow. This has been also found for the case of BAE and CFIE applied to the scalar Helmholtz equation [36]. It is also a numerical evidence that the BAE method is very precise. This is mainly due to lack of numerical integrals and that most of the steps of the method are exact, ending with a set of algebraic equations. As a result, the fictitious eigenfrequency only has effect at the exact frequency. On the contrary, in BEM the numerical integrals on the boundary introduce some errors. This creates a frequency band around the fictitious eigenfrequency where the solution loses the accuracy. This is clearly shown in [32] and it is also mentioned that the effect of fictitious eigenfrequencies is something not easy to find but that can always appear. There [32], a procedure is proposed to understand whether the computation is affected or not by one of these eigenfrequencies and whether some of the available remedies must be applied. According to the values of frequency shown in [32], the frequency band width of BEM is considerably much wider than this of BAE (illustrated in Table 3).

In any case, the CFIE formulation works well and it damps numerically the fictitious eigenfrequencies. It also helps in order to have the matrix condition number under control. This can be very useful in two different applications of the method. On the one hand to big problems or problems that fall in the mid-frequencies and has large modal density. In this situation, the effect of fictitious eigenfrequencies is more important. On the other hand, sometimes it is costly to compute the discrete Green's function, and it is a good idea to evaluate them by means of some approximation like asymptotic formulas [28]. This would introduce some lack of precision that would play the role of integration errors in BEM and make the effect of fictitious eigenfrequencies evident in a wider frequency band (and consequently CFIE more necessary).

\subsection{Interior problem: one-dimensional $\mathrm{P}$-wave}

The solution of interior elastodynamic problems is not the main goal of the proposed technique. Only in some cases having very large domains (with respect to the solution wavelength), BAE can be competitive with the other techniques such as FEM. However, at least one interior problem can be considered to explore the behaviour of BAE. The problem is solved with BAE and no error due to the geometry approximation is expected (the domain is rectangular shaped). The interior problem has eigenfrequencies (which have physical meaning and are not fictitious). It is difficult to find exterior problems with grid-shaped boundaries and available exact (analytical) solution, while for the interior problem of Fig. 11 there exists one.

$\mathrm{L}_{\mathrm{x}}$

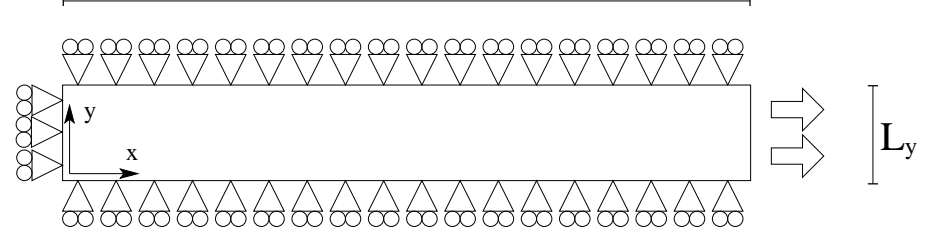

Figure 11: Rectangle with imposed one-dimensional displacement. 
Consider an homogeneous and isotropic elastic rectangle with boundary conditions chosen in order to generate a one-dimensional wave. The boundary conditions are

$$
\begin{array}{rr}
u^{x}=0 & \text { for } x=0 \\
u^{y}=0 & \text { for } y=0 \\
u^{y}=0 & \text { for } y=L_{y} \\
u^{x}=\Delta & \text { for } x=L_{x}
\end{array}
$$

The analytical solution of this problem is

$$
\begin{aligned}
& u^{x}(x, y)=\frac{\Delta}{\sin \left(L_{x} \sqrt{k_{P}}\right)} \sin \left(x \sqrt{k_{P}}\right) \quad \text { with } k_{P}=\frac{\rho \omega^{2}}{\lambda+2 \mu} \\
& u^{y}(x, y)=0
\end{aligned}
$$

The solution is a one-dimensional $\mathrm{P}$-wave with wavenumber $k_{P}$. The material properties are those of Table 1 and the dimensions of the rectangular domain $L_{x}=20 \mathrm{~m}$ and $L_{y}=4 \mathrm{~m}$ (the dimension of $L_{y}$ is not important if there are no transverse waves). With these values, the first eigenfrequencies of the problem are at $\omega / 2 \pi=97.3 \mathrm{~Hz}$, 194.6 Hz, 291.9 Hz, 389.2 Hz, 486.6 Hz and 583.8 Hz. They correspond to standing P-waves.

The results are shown in Fig. 12. When studying the effect of the grid size for a constant frequency (Fig. 12(a)), the trend is very similar to those of linear triangular or quadrangular elements in elasticity. The slope $\log _{10}(e) / \log _{10}(h)$ is close to 2 . It can be concluded from Fig. 12(b) that a global trend of the error with respect to the dimensionless wave number of the solution is $\log _{10}(e) / \log _{10}\left(k_{P} h\right) \approx 3$.

This result is largely modified around the eigenfrequencies of the problem. It is a direct consequence of the small difference between the wavenumber of the numerical solution and the exact wavenumber (k-singularity, [5]). This phenomenon implies also a small difference in the frequency value between the eigenfrequencies of the exact solution and the numerical ones. In addition, the resonances have a very narrow band width influence due to the lack of damping. All these causes that a calculation at a frequency which is close to the eigenfrequency zone can have a resonating numerical solution and non-resonating exact solution or vice-versa (ideally, in a numerical simulation free of error, both solutions would be resonating at the same frequency). One of the two solutions (numerical or exact) is amplified while the other not, which leads to a larger numerical error (with respect of a computation at a frequency not close to the eigenfrequency zone). As a result, Fig. 12(c) shows error peaks around the eigenfrequencies.

\section{Concluding remarks}

The main conclusions drawn from the proposed method and numerical examples are as follows:

1. A numerical method based on the boundary algebraic equations is presented. Its capabilities are demonstrated by several numerical examples. One can see 


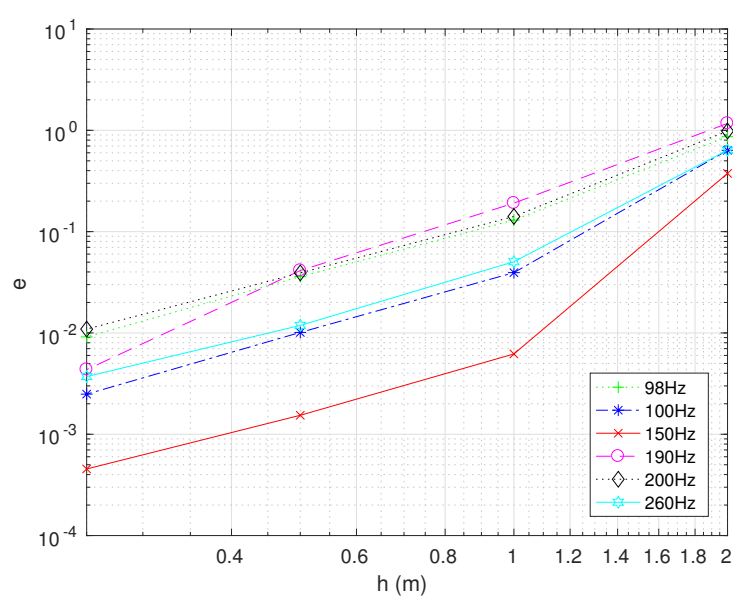

(a)

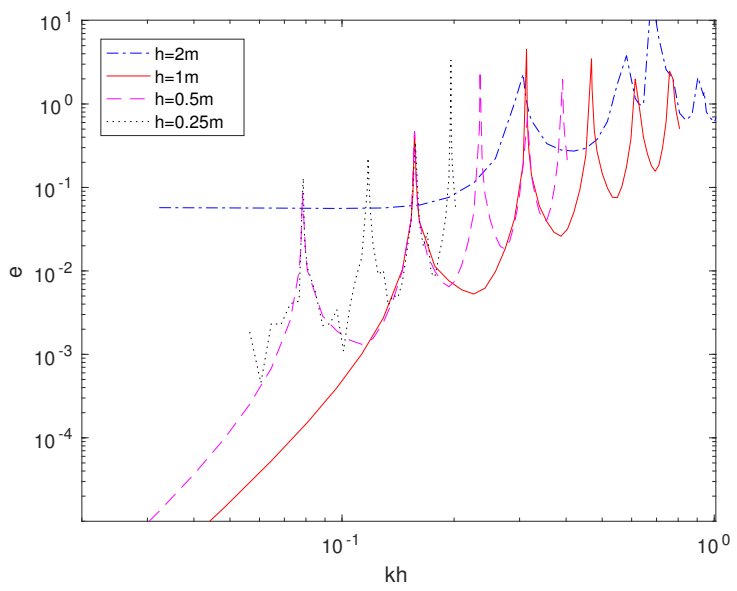

(b)

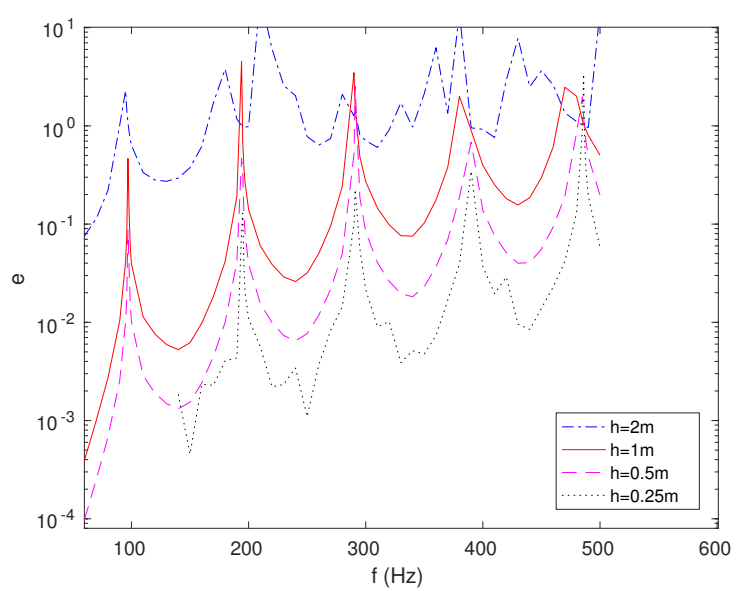

(c)

Figure 12: Rectangle with imposed one-dimensional displacement. Dependence of the relative error: (a) on the grid size for several frequencies; (b) on the dimensionless wavenumber for a constant grid size; (c) on the frequency for a constant grid size.

that it is a valid technique for solving elastodynamic problems in the frequency domain.

2. Two discrete analogues of boundary integral equations are provided: the standard one Eq. (16) (valid for interior and exterior problems), and the CFIE equivalent Eq. (20) (this second one has only sense for exterior problems).

3. Eq. (20) is free of the fictitious eigenfrequencies for the exterior problems and also keeps the condition number of the system matrix more or less constant. However, Eq. (16) provides also good results because, contrary to what happens in BEM, the effect of fictitious eigenfrequencies in BAE is extremely concentrated around specific frequency. It is shown in Table 3 that the influence zone can be less than $10^{-9} \mathrm{~Hz}$ for the particular example. This is also an evidence of the precision of the presented formulation.

4. BAE can be successfully combined with FEM. This combination can be used to 
reduce the geometry error in those problems where the solid boundary is curved and is badly approximated by a regular mesh. A thin layer of finite elements overcomes this difficulty.

5. The convergence rate of BAE is similar to that of the linear FEM used to derive operator $L$.

BAE is an adequate technique that can be used to succesfully solve the elastodynamic problem. Although the current research is performed in the framework of two-dimensional plane strain, the doors are open to extend the method to other problems.

\section{A Example of linear operator}

Here we present an example of linear operator $L$ and how it can be truncated in order to describe the grid-shaped geometry. This complements the previous sections where the consideration has been held for a generic linear operator. The operator detailed here is based on bilinear quadrilateral finite elements (FEM) for plane strain [13] (two-dimensional elastodynamics in the frequency domain). It is the operator used in the numerical examples. However, any linear representation that satisfies the structure of the linear operator in Eq. (4), the symmetry conditions in Eq. (5) and allows a proper truncation representing the boundary conditions, would be valid.

Another possibility (not studied here) is to define a linear operator based on finite differences (FD). The truncation of the operator is in that case more laborious. See for example [33, 40] where both the FD stencil and the most usual boundary conditions in elastodynamics are defined. In all cases, the procedure described in Section 2.5 must be used to compute the discrete tensor Green's function.

The FEM-based operator with a lumped mass matrix can be defined as follows:

$$
L^{\prime}[u]_{j}^{v} \equiv \sum_{q \in \Omega^{\prime}} \beta_{j, q}^{\prime v, \eta} u_{q}^{\eta}, \quad j \in \Omega^{\prime}, \quad v, \eta=x, y,
$$

with

$$
\beta_{j, q}^{\prime v, \eta}=-\lambda \beta_{\lambda j, q}^{\prime v, \eta}-\mu \beta_{\mu_{j, q}}^{v, \eta}+\rho \omega^{2} h^{2} \delta_{v, \eta}
$$

The loop about the nodes $q$ surrounding and including the node $j\left(\odot_{j}\right)$ is expressed here in terms of a $3 \times 3$ matrix

$$
u_{q}^{\eta}=\left[\begin{array}{ccc}
u^{\eta} j_{x}+1, j_{y}-1 & u_{j_{x}+1, j_{y}}^{\eta} & u_{j_{x}+1, j_{y}+1}^{\eta} \\
u_{j_{x}, j_{y}-1}^{\eta} & u_{j_{x}, j_{y}}^{\eta} & u_{j_{x}, j_{y}+1}^{\eta_{2}} \\
u_{j_{x}-1, j_{y}-1}^{\eta} & u_{j_{x}-1, j_{y}} & u^{\eta} j_{j_{x}-1, j_{y}+1}
\end{array}\right], \quad q \in \odot_{j}, \quad \eta=x, y
$$

The operation $\beta_{j, q}^{\prime v, \eta} u_{q}^{\eta}$ in performed here as the double-dot product between the $3 \times 3$ matrices $\beta_{\lambda}^{\prime}{ }_{\lambda}^{v, \eta}$ and $u^{\eta}$.

The values of the coefficients in the matrices $\beta_{\lambda j, q}^{\prime v, \eta}$ and $\beta_{\mu_{j, q}^{\prime}}^{v, \eta}$ can be found by direct assembly of elemental matrices of bilinear quadrilateral elements for plane strain. They need to be particularised as squares of side $h$. 


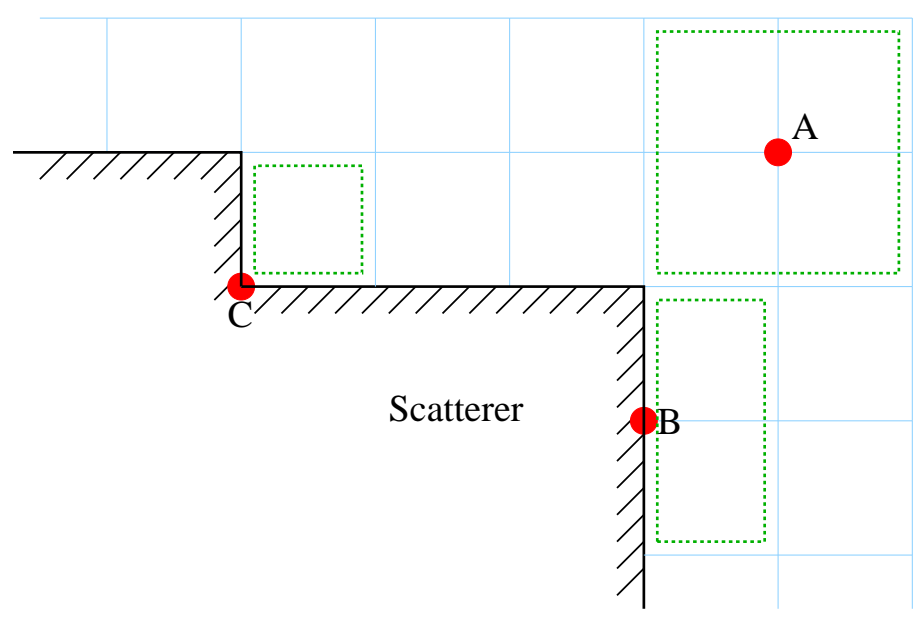

Figure 13: Example of three nodes of the grid where the operator $L^{\prime}$ is defined: A - Field node, assembly of four quadrilateral elements; B - Side node, assembly of two quadrilateral elements; C - Interior corner node, assembly of one quadrilateral element.

Field node (A in Fig. 13) The operator for a node in the field is the result of the assembly of a patch of four elements. See the node A in Fig. 13). In that case the operators $L^{\prime}$ and $L$ are the same. This form of the operator is the one required in order to compute the tensor discrete Green's function (Section 2.5). The value of the coefficients are

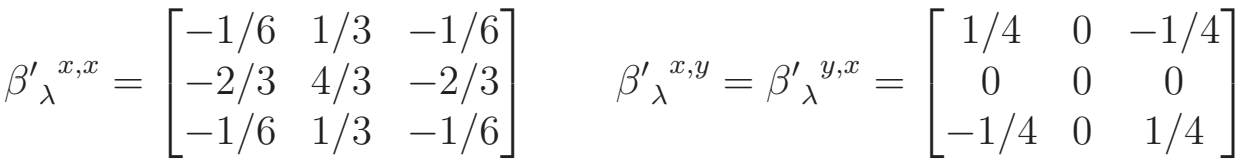

$$
\begin{aligned}
& \beta_{\lambda}^{\prime}{ }_{\lambda}^{y, x}=\beta_{\lambda}^{\prime}{ }^{x, y} \\
& {\beta_{\lambda}^{\prime}}_{\lambda}^{y, y}=\left[\begin{array}{ccc}
-1 / 6 & -2 / 3 & -1 / 6 \\
1 / 3 & 4 / 3 & 1 / 3 \\
-1 / 6 & -2 / 3 & -1 / 6
\end{array}\right] \\
& \beta_{\mu}^{\prime x x}=\left[\begin{array}{ccc}
-1 / 2 & 0 & -1 / 2 \\
-1 & 4 & -1 \\
-1 / 2 & 0 & -1 / 2
\end{array}\right] \\
& \beta_{\mu}^{\prime}{ }^{x y}=\beta_{\mu}^{\prime}{ }^{y x}=\left[\begin{array}{ccc}
1 / 4 & 0 & -1 / 4 \\
0 & 0 & 0 \\
-1 / 4 & 0 & 1 / 4
\end{array}\right] \\
& \beta_{\mu}^{\prime y y}=\left[\begin{array}{ccc}
-1 / 2 & -1 & -1 / 2 \\
0 & 4 & 0 \\
-1 / 2 & -1 & -1 / 2
\end{array}\right]
\end{aligned}
$$

Side node (B in Fig. 13) An example of the truncation of the operator $L$ in order to obtain $L^{\prime}$ is the side node B in Fig. 13. It is obtained by assembly of two square elements. It is important to note that FEM-based operator has the advantage that the truncation has physical meaning: a boundary free of forces.

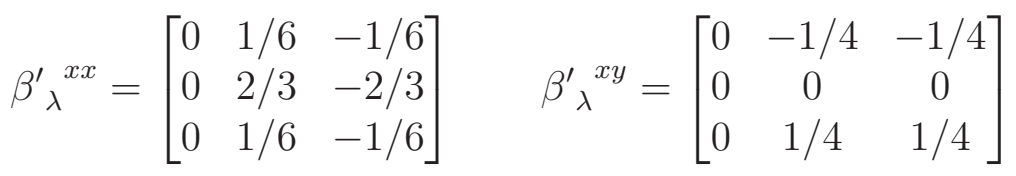




$$
\begin{aligned}
& \beta_{\lambda}^{\prime y x}=\left[\begin{array}{ccc}
0 & 1 / 4 & -1 / 4 \\
0 & 0 & 0 \\
0 & -1 / 4 & 1 / 4
\end{array}\right] \beta_{\lambda}^{\prime y y}=\left[\begin{array}{ccc}
0 & -1 / 3 & -1 / 6 \\
0 & 2 / 3 & 1 / 3 \\
0 & -1 / 3 & -1 / 6
\end{array}\right] \\
& {\beta^{\prime}}_{\mu}^{x x}=\left[\begin{array}{ccc}
0 & 0 & -1 / 2 \\
0 & 2 & -1 \\
0 & 0 & -1 / 2
\end{array}\right] \quad \beta_{\mu}^{\prime}{ }^{x y}=\left[\begin{array}{ccc}
0 & 1 / 4 & -1 / 4 \\
0 & 0 & 0 \\
0 & -1 / 4 & 1 / 4
\end{array}\right] \\
& \beta_{\mu}^{\prime}{ }^{y x}=\left[\begin{array}{ccc}
0 & -1 / 4 & -1 / 4 \\
0 & 0 & 0 \\
0 & 1 / 4 & 1 / 4
\end{array}\right] \beta_{\mu}^{\prime}{ }^{y y}=\left[\begin{array}{ccc}
0 & -1 / 2 & -1 / 2 \\
0 & 2 & 0 \\
0 & -1 / 2 & -1 / 2
\end{array}\right]
\end{aligned}
$$

Interior corner node ( $\mathrm{C}$ in Fig. 13) Finally, an interior corner node, which is obtained by assembly of only one element is shown:

$$
\begin{aligned}
& {\beta^{\prime}{ }_{\lambda}{ }^{x x}}^{x}=\left[\begin{array}{ccc}
0 & 1 / 6 & -1 / 6 \\
0 & 1 / 3 & -1 / 3 \\
0 & 0 & 0
\end{array}\right] \quad{\beta^{\prime}{ }_{\lambda}{ }^{x y}}=\left[\begin{array}{ccc}
0 & -1 / 4 & -1 / 4 \\
0 & 1 / 4 & 1 / 4 \\
0 & 0 & 0
\end{array}\right]
\end{aligned}
$$

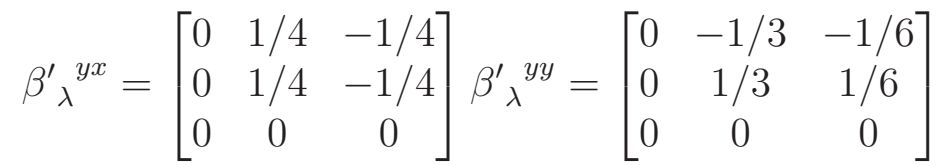

$$
\begin{aligned}
& \beta_{\mu}^{\prime x x}=\left[\begin{array}{ccc}
0 & 0 & -1 / 2 \\
0 & 1 & -1 / 2 \\
0 & 0 & 0
\end{array}\right] \quad \beta_{\mu}^{\prime}{ }^{x y}=\left[\begin{array}{ccc}
0 & 1 / 4 & -1 / 4 \\
0 & 1 / 4 & -1 / 4 \\
0 & 0 & 0
\end{array}\right] \\
& \beta_{\mu}^{\prime}{ }_{\mu}^{y x}=\left[\begin{array}{ccc}
0 & -1 / 4 & -1 / 4 \\
0 & 1 / 4 & 1 / 4 \\
0 & 0 & 0
\end{array}\right] \beta^{\prime}{ }_{\mu}{ }^{y y}=\left[\begin{array}{ccc}
0 & -1 / 2 & -1 / 2 \\
0 & 1 & 0 \\
0 & 0 & 0
\end{array}\right]
\end{aligned}
$$

Force vector due to a uniformly distributed force per unit volume A similar formalism as in Eq. (53) can be used if an external force per unit volume $\left(f_{v}^{x}, f_{v}^{y}\right)$ needs to be considered. This can be included in the force term of Eq. (7) by means of the following discretisation, which is also based on the assembly of square finite elements for plane stress:

$$
f_{j}^{v} \equiv \sum_{q \in \Omega^{\prime}} \frac{h^{2}}{4} \phi_{j, q}^{\prime v, \eta} f_{v q}^{\eta}, \quad j \in \Omega^{\prime}, \quad v, \eta=x, y,
$$

The known values of the force per unit volume can be displayed in matrix form as

$$
f_{v q}^{\eta}=\left[\begin{array}{ccc}
f_{v j_{x}+1, j_{y}-1}^{\eta} & f_{v j_{x}+1, j_{y}}^{\eta} & f_{v j_{x}+1, j_{y}+1}^{\eta} \\
f_{v j_{x}, j_{y}-1}^{\eta} & f_{v j_{x}, j_{y}}^{\eta} & f_{v j_{x}, j_{y}+1}^{\eta} \\
f_{v j_{x}-1, j_{y}-1}^{\eta} & f_{v j_{x}-1, j_{y}}^{\eta} & f_{v j_{x}-1, j_{y}+1}^{\eta}
\end{array}\right], \quad q \in \odot_{j}, \quad \eta=x, y
$$

and the force term $f_{j}^{v}$ is build in an analogous way as in Eq. (53) by means of the double-dot product between the $3 \times 3$ matrices $\phi^{\prime v, \eta}$ and $f_{v_{q}}^{\eta}$. The definition of $\phi^{\prime v, \eta}$ 
for a field node (A in Fig. 13) is

$$
\phi^{\prime x x}=\phi^{\prime y y}=\left[\begin{array}{lll}
1 & 2 & 1 \\
2 & 4 & 2 \\
1 & 2 & 1
\end{array}\right] \quad \phi^{\prime x y}=\phi^{\prime y x}=\left[\begin{array}{lll}
0 & 0 & 0 \\
0 & 0 & 0 \\
0 & 0 & 0
\end{array}\right]
$$

for a side node (B in Fig. 13) is

$$
\phi^{\prime x x}=\phi^{\prime y y}=\left[\begin{array}{lll}
0 & 1 & 1 \\
0 & 2 & 2 \\
0 & 1 & 1
\end{array}\right] \quad \phi^{\prime x y}=\phi^{\prime y x}=\left[\begin{array}{lll}
0 & 0 & 0 \\
0 & 0 & 0 \\
0 & 0 & 0
\end{array}\right]
$$

and finally for an interior corner node (C in Fig. 13)

$$
\phi^{\prime x x}=\phi^{\prime y y}=\left[\begin{array}{lll}
0 & 1 & 1 \\
0 & 1 & 1 \\
0 & 0 & 0
\end{array}\right] \quad \phi^{\prime x y}=\phi^{\prime y x}=\left[\begin{array}{lll}
0 & 0 & 0 \\
0 & 0 & 0 \\
0 & 0 & 0
\end{array}\right]
$$

\section{Acknowledgements}

The authors acknowledge the Euro-Russian Academic Network-Plus program (grant number 2012-2734/001-001-EMA2). J. Poblet-Puig from the LaCàN research group is grateful for the sponsorship/funding received from Generalitat de Catalunya (Grant number 2014-SGR-1471). A.V. Shanin has been supported by RSF grant 14-22-00042.

\section{References}

[1] J.D. Achenbach. Wave Propagation in Elastic Solids. Applied mathematics and Mechanics, vol. 16. North-Holland Publishing Company, 1975.

[2] A. Ali and Ch. Rajakumar. The boundary element method: Applications in sound and vibration. CRC Press, 2004.

[3] D.E. Beskos. Boundary element methods in dynamic analysis. Applied Mechanics Reviews, 40(1):1-23, 1987.

[4] H. S. Bhat and B. Osting. Diffraction on the two-dimensional square lattice. SIAM J. Appl. Math., 70(5):1389-1406, 2009.

[5] P. Bouillard and F. Ihlenburg. Error estimation and adaptivity for the finite element method in acoustics: 2D and 3D applications. Comput. Methods Appl. Mech. Eng., 176(1-4):147-163, 1999.

[6] A.J. Burton and G.F. Miller. Application of integral equation methods to numerical solution of some exterior boundary-value problems. Proc. R. Soc. A-Math. Phys. Eng. Sci., 323(1553):201-\&, 1971. 
[7] S. Chaillat, M. Bonnet, and J.-F. Semblat. A new fast multi-domain BEM to model seismic wave propagation and amplification in 3-D geological structures. Geophysical Journal International, 177(2):509-531, 2009.

[8] M. Darbas and F. Le Louër. Well-conditioned boundary integral formulations for high-frequency elastic scattering problems in three dimensions. Mathematical Methods in the Applied Sciences, 38(9):1705-1733, 2015.

[9] A. Dijckmans, A. Ekblad, A. Smekal, G. Degrande, and G. Lombaert. Efficacy of a sheet pile wall as a wave barrier for railway induced ground vibration. Soil Dynamics and Earthquake Engineering, 84:55-69, 2016.

[10] J. Dominguez. Boundary elements in dynamics. Wit Press, 1993.

[11] J.M. Doyle. Radiation conditions in elasticity. Zeitschrift für angewandte Mathematik und Physik ZAMP, 16(4):527-531, 1965.

[12] A.C. Eringen and E.S. Suhubi. Elastodynamics. Academic Press, 1974.

[13] H. P. Gavin. Structural element stiffness, mass, and damping matrices. Technical report, Department of Civil and Environmental Engineering, Duke University, 2014.

[14] C. Geuzaine and J.-F. Remacle. Gmsh: a three-dimensional finite element mesh generator with built-in pre- and post-processing facilities. Int. J. Numer. Meth. Engng., 11(79):1309-1331, 2009.

[15] D. Gross and Ch. Zhang. Wave propagation in damaged solids. International Journal of Solids and Structures, 29(14):1763-1779, 1992.

[16] L. Guan and A. Norris. Elastic wave scattering by rectangular cracks. International Journal of Solids and Structures, 29(12):1549-1565, 1992.

[17] T. Hagstrom. Radiation boundary conditions for the numerical simulation of waves. Acta numerica, 8:47-106, 1999.

[18] P. Jean, C. Guigou, and M. Villot. A 2.5 D BEM model for ground-structure interaction. Building Acoustics, 11(3):157-173, 2004.

[19] D.S. Jones. An exterior problem in elastodynamics. In Math. Proc. Camb. Phil. Soc, volume 96, pages 173-82. Cambridge Univ Press, 1984.

[20] D.S. Jones. Boundary integrals in elastodynamics. IMA journal of applied mathematics, 34(1):83-97, 1985.

[21] E. Kausel. Fundamental solutions in elastodynamics: a compendium. Cambridge University Press, 2006.

[22] K. Kiriaki. A uniquely solvable integral equation for the neumann problem in linearelasticity. Applicable Analysis, 73(3-4):379-392, 1999. 
[23] S. Kobayashi and N. Nishimura. On the indeterminancy of BIE solutions for the exterior problems of time-harmonic elastodynamics and incompressible elastostatics(boundary integral equation). Boundary element methods in engineering, pages 282-296, 1982.

[24] J Koko. Vectorized Matlab codes for linear two-dimensional elasticity. Scientific Programming, 15(3):157-172, 2007.

[25] P Langer, M Maeder, C Guist, M Krause, and S Marburg. More than six elements per wavelength: The practical use of structural finite element models and their accuracy in comparison with experimental results. Journal of Computational Acoustics, page 1750025, 2017.

[26] G.D. Manolis. A comparative study on three boundary element method approaches to problems in elastodynamics. Int. J. Numer. Meth. Engng., 19(1):7391, 1983.

[27] S. Marburg. The Burton and Miller method: Unlocking another mystery of its coupling parameter. Journal of Computational Acoustics, 24(01):1550016, 2016.

[28] P. A. Martin. Discrete scattering theory: Green's function for a square lattice. Wave Motion, 43(7):619-629, 2006.

[29] P. G. Martinsson and G. J. Rodin. Boundary algebraic equations for lattice problems. Proc. R. Soc. A-Math. Phys. Eng. Sci., 465(2108):2489-2503, 2009.

[30] M. Medvinsky, S. Tsynkov, and E. Turkel. The method of difference potentials for the Helmholtz equation using compact high order schemes. Journal of Scientific Computing, 53:150-193, 2012.

[31] G. Müller. Theory of Elastic Waves. Scientific Technical Report 2007 : GeoForschungsZentrum Potsdam. GFZ, 2007.

[32] N. Nishimura. Applications of the Boundary Integral Equation Methods to Solid Mechanics. PhD thesis, Kyoto University, 1988.

[33] J.A. Pérez-Ruiz, F. Luzón, and A. García-Jerez. Simulation of an irregular free surface with a displacement finite-difference scheme. Bulletin of the Seismological Society of America, 95(6):2216-2231, 2005.

[34] J. Poblet-Puig and A.V. Shanin. A combined FEM/BEM discrete numerical method for solving external scattering problems in acoustics. In Computational Experiment in Aeroacoustics, pages 126-127, Sep 2016.

[35] J. Poblet-Puig, V.Yu. Valyaev, and A.V. Shanin. Boundary element method based on preliminary discretization. Mathematical Models and Computer Simulations, 6(2):172-182, 2014.

[36] J. Poblet-Puig, V.Yu. Valyaev, and A.V. Shanin. Suppression of spurious frequencies in scattering problems by means of boundary algebraic and combined field equations. Journal of Integral Equations and Applications, 27:233-274, 2015. 
[37] L. Pyl, D. Clouteau, and G. Degrande. A weakly singular boundary integral equation in elastodynamics for heterogeneous domains mitigating fictitious eigenfrequencies. Engineering Analysis with Boundary Elements, 28(12):1493-1513, 2004.

[38] M.B. Radosz. The principles of limit absorption and limit amplitude for periodic operators. PhD thesis, Karlsruher Inst. für Technologie, Diss., 2010, 2009.

[39] M. Rezayat, D.J. Shippy, and F.J. Rizzo. On time-harmonic elastic-wave analysis by the boundary element method for moderate to high frequencies. Computer methods in applied mechanics and engineering, 55(3):349-367, 1986.

[40] B. Ruud and S. Hestholm. 2D surface topography boundary conditions in seismic wave modelling. Geophysical Prospecting, 49(4):445-460, 2001.

[41] H.A. Schenck. Improved integral formulation for acoustic radiation problems. $J$. Acoust. Soc. Am., 44(1):41-58, 1968.

[42] B.L. Sharma. Diffraction of waves on square lattice by semi-infinite crack. SIAM Journal on Applied Mathematics, 75(3):1171-1192, 2015.

[43] B.L. Sharma. Near-tip field for diffraction on square lattice by crack. SIAM Journal on Applied Mathematics, 75(4):1915-1940, 2015.

[44] B.L. Sharma. Continuum limit of discrete Sommerfeld problems on square lattice. Sädhanā, pages 1-16, 2017.

[45] R.N. Simpson, M.A. Scott, M. Taus, D. C Thomas, and H. Lian. Acoustic isogeometric boundary element analysis. Computer Methods in Applied Mechanics and Engineering, 269:265-290, 2014.

[46] D.M. Tracey. Finite elements for determination of crack tip elastic stress intensity factors. Engineering Fracture Mechanics, 3(3):255-265, 1971.

[47] B.E. Treeby and J. Pan. A practical examination of the errors arising in the direct collocation boundary element method for acoustic scattering. Engineering Analysis with Boundary Elements, 33(11):1302-1315, 2009.

[48] I. Tsukerman. A Singularity-Free Boundary Equation Method for Wave Scattering. IEEE Trans. Antennas Propag., 59(2):555-562, 2011.

[49] B.R. Vainberg. Principles of radiation, limit absorption and limit amplitude in the general theory of partial differential equations. Russian Mathematical Surveys, 21(3):115-193, 1966.

[50] W.H. Woodward, S. Utyuzhnikov, and P. Massin. On the application of the method of difference potentials to linear elastic fracture mechanics. International Journal for Numerical Methods in Engineering, 103(10):703-736, 2015.

[51] O.C. Zienkiewicz and R.L. Taylor. The finite element method (Fifth edition). 